DocDB-7923

May 13, 2011

\title{
MINOS+
}

\section{A Proposal to FNAL to run MINOS with the medium energy NuMI beam}

\section{The MINOS+ Collaboration}

\author{
G. Tzanakos \\ University of Athens, Athens, Greece \\ M. Bishai, M. Diwan \\ Brookhaven National Laboratory, Upton, New York 11973, USA \\ C. O. Escobar R. A. Gomes P. Gouffon
}

Universidade Estadual de Campinas, IFGW-UNICAMP, CP 6165, 13083-970, Campinas, SP, Brazil

Instituto de Física, Universidade Federal de Goiás, CP 131, 74001-970, Goiânia, GO, Brazil

Instituto de Física, Universidade de São Paulo, CP 66318, 05315-970, São Paulo, SP, Brazil

A. Blake, M. Thomson

Cavendish Laboratory, University of Cambridge, Madingley Road, Cambridge CB3 OHE, UK

R. B. Patterson

Lauritsen Laboratory, California Institute of Technology, Pasadena, California 91125, USA

P. Adamson, S. Childress, P. Lucas, A. Kreymer, B. Rebel, B. Pahlka, R. Plunkett ${ }^{1}$

Fermi National Accelerator Laboratory, Batavia, Illinois 60510, USA

C. White

Physics Division, Illinois Institute of Technology, Chicago, Illinois 60616, USA

G. Mills, Z. Pavlović

Los Alamos National Laboratory, Los Alamos, New Mexico 87545, USA

D. Cronin-Hennessy, M. Marshak, G. Pawloski

University of Minnesota, Minneapolis, Minnesota 55455, USA

A. Habig

Department of Physics, University of Minnesota - Duluth, Duluth, Minnesota 55812, USA

S. Swain

National Institute of Scientific Education and Research, Bhubaneswar, Orissa, India.

M. C. Sanchez

Department of Physics and Astronomy, Iowa State University, Ames, Iowa 50011 USA

N. Tagg

Otterbein College, Westerville, Ohio 43081, USA

\footnotetext{
${ }^{1}$ Co-spokesperson
} 
G. Barr, J. de Jong, A. Weber

Subdepartment of Particle Physics, University of Oxford, Oxford OX1 3RH, UK

Z. Isvan, D. Naples

Department of Physics and Astronomy, University of Pittsburgh, Pittsburgh, Pennsylvania 15260, USA

S. Mishra, C. Rosenberg

Department of Physics and Astronomy, University of South Carolina, Columbia, South Carolina 29208, USA

S. G. Wojcicki

Department of Physics, Stanford University, Stanford, California 94305, USA

E. Falk, J. Hartnell

Department of Physics and Astronomy, University of Sussex, Falmer, Brighton BN1 9QH, UK

R. Mehdiyev, K. Lang, J. L. Ritchie

Department of Physics, University of Texas at Austin, 1 University Station C1600, Austin, Texas 78712, USA

A. Mann, J. Schneps, H. Gallagher

Physics Department, Tufts University, Medford, Massachusetts 02155, USA

J. Evans, A. Holin, R. Nichol, J. Thomas ${ }^{2}$

Department of Physics and Astronomy, University College London, Gower Street, London WC1E 6BT, UK

K. Grzelak

Department of Physics, Warsaw University, Hoża 69, PL-00-681 Warsaw, Poland

M. Kordosky, J. Nelson, P. Vahle

Department of Physics, College of William \& Mary, Williamsburg, Virginia 23187, USA

\section{Executive Summary}

This is a proposal to continue to expose the two MINOS detectors to the NuMI muon neutrino beam for three years starting in 2013. The medium energy setting of the NuMI beam projected for $\mathrm{NO} \nu \mathrm{A}$ will deliver about $18 \times 10^{20}$ protons-on-target during the first three years of operation. This will allow the MINOS Far Detector to collect more than 10,000 charged current muon neutrino events in the 4-10 GeV energy range and provide a stringent test for non-standard neutrino interactions, sterile neutrinos, extra dimensions, neutrino time-of-flight, and perhaps more. In addition there will be more than 3,000 neutral current events which will be particularly useful in extending the sterile neutrino search range.

\footnotetext{
${ }^{2}$ Co-spokesperson
} 


\section{Introduction}

It was slightly more than a decade ago that the consensus began to emerge that neutrinos are massive and that they oscillate, providing the first ever clear evidence of physics beyond the Standard Model. Neutrino oscillations are now widely accepted as the dominant mechanism behind the observed neutrino anomalies of the late 20th century $[1,2,9]$. These phenomena are governed by two neutrino mass splittings, $\Delta m_{32}^{2}$ and $\Delta m_{21}^{2}$, and the $3 \times 3$ PMNS mixing matrix [4] which can be parametrized by three mixing angles $\theta_{i j}, \mathrm{ij}=12,23,13$, and a CP-violating phase $\delta_{c p}$. The best measurement of $\left|\Delta m_{32}^{2}\right|$, or the atmospheric mass splitting, has recently been published by the MINOS experiment [2]. MINOS observes oscillations as an energy-dependent disappearance of muon neutrino charged current (CC) interactions between two detectors [5] separated by a distance of $734 \mathrm{~km}$. Using data from an exposure of $7.25 \times 10^{20}$ protons-on-target (POT) in the lowenergy setting of the NuMI beam at Fermilab, MINOS has found $\left|\Delta m_{32}^{2}\right|=\left(2.32_{-0.08}^{+0.12}\right) \times 10^{-3} \mathrm{eV}^{2}$ and $\sin ^{2}\left(2 \theta_{23}\right)>0.90(90 \%$ C.L. $)$, achieving a level of about $5 \%$ precision on $\left|\Delta m_{32}^{2}\right|$. The simple two-flavor oscillation model describes the energy dependence of the survival probability, while two other hypotheses, pure neutrino decay [6] and pure quantum decoherence [7] are excluded at 7 and 9 standard deviations, respectively. The best measurement of the anti-neutrino oscillation parameters have also been recently published by MINOS [8], yielding $\Delta \overline{\mathrm{m}}^{2}=3.36_{-0.40}^{+0.46} \mathrm{eV}^{2}$ and $\sin ^{2} 2 \bar{\theta}_{23}=0.86 \pm_{-0.12}^{+0.11}$, but the agreement between these and the neutrino parameters is only at the $2 \%$ level. The best constraints on the atmospheric mixing angle are provided by the SuperKamiokande experiment [9].

Despite increasingly precise measurements of some of the oscillation parameters, the field of neutrino oscillations is still in its relative infancy. While the magnitudes of the mass splittings and two of the mixing angles have been measured, the mixing angle $\theta_{13}$ and the CP violating phase $\delta_{c p}$ remain unknown. Furthermore, the sign of $\Delta m_{32}^{2}$, or the mass hierarchy, is also unknown. While some fraction of the world's neutrino community are searching for evidence of anomalous neutrino behaviour both at large and small values of $\mathrm{L} / \mathrm{E}$, the majority of world-wide effort in neutrino physics is now being directed towards measuring these last two parameters. The two major experiments with this goal, T2K and NOvA, will both require neutrino energy spectra tightly peaked right at the first oscillation minimum. NOvA achieves this requirement by running the NuMI beam in the medium energy setting, and by using the off-axis strategy. During the NOvA era, the MINOS experiment will be in a unique position to significantly refine our knowledge of neutrino oscillation properties by measuring the shape of the oscillation probability at energies above the first minimum, until now considered less important than the region most affected by oscillation. Figure 1 shows the MINOS CC energy spectrum from the latest measurement and illustrates the level of statistical uncertainty, which is presently the limiting factor in the uncertainty of the mass splitting. Clearly, additional events above the low energy beam focusing peak at $3 \mathrm{GeV}$ would improve the determination of the shape of the oscillation probability, which could be essential in further testing of the oscillation theory. The event rate between $4-10 \mathrm{GeV}$ would be more than 5 times that in the low energy beam with a factor 7 more neutrinos being directed along the beam axis than off axis.

In this proposal, the case is made for running MINOS during the NOvA era, to be referred to as the MINOS+ experiment. It offers unprecedented and unique opportunities to explore physics beyond the three-neutrino mixing model. MINOS+ will further exploit the MINOS-NuMI potential. Highstatistics neutrino data can be collected in the Far Detector to test existence of non-standard neutrino interactions, sterile neutrinos, extra dimensions, neutrino life-time, as well as increasing 


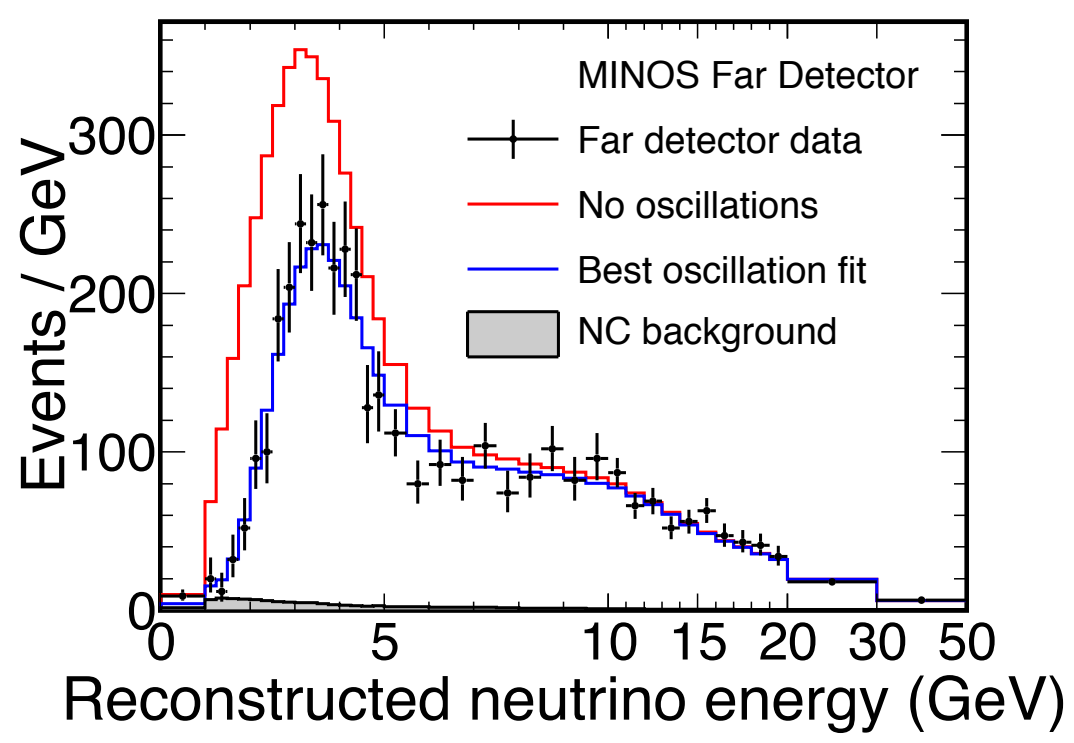

Figure 1: The measured neutrino energy spectrum from the MINOS experiment.

accuracy on the standard parameters. The MINOS+ experiment will be in a very fortunate position of measuring a green-field region of energy and precision where these very disparate effects could be seen. Such an opportunity comes very rarely. ${ }^{3}$

\section{MINOS energy spectrum}

Using the NuMI-NO $\nu$ A beam prediction ${ }^{4}$ and assuming a yearly intensity of $0.6 \times 10^{21}$ P.O.T, it is estimated that about $3000 \mathrm{CC}$ and $1200 \mathrm{NC}$ events will be registered in the MINOS detector between $4-10 \mathrm{GeV}$ per year. Figure 2 (left) shows the NuMI NO $\nu \mathrm{A}$ spectrum in red and the on-axis beam to be measured in MINOS+ in black [10]. The mean energy of the on-axis events will be around $7.5 \mathrm{GeV}$, in an energy region much better suited to the segmentation of the MINOS Far Detector than the NuMI MINOS LE beam. In this region presently we have an uncertainty of about $25 \%$ per $\mathrm{GeV}$ whereas two years of running in the NUMI-NO $\nu \mathrm{A}$ beam would produce error bars of $5 \%$ per $0.5 \mathrm{GeV}$ in the MINOS FD. In total, about $4800 \mathrm{CC}$ and $1600 \mathrm{NC}$ events per year will be produced in the MINOS detector between 1-80 GeV, more than twice the total number of events collected by MINOS over the first five years. The ratio of oscillated to un-oscillated spectra are shown in Figure 2 (right) for a three year run period compared to the present precision of the

\footnotetext{
${ }^{3}$ The few examples include a) discovery of b quark made possible by new energy realm (but imagined by Kobayashi and Maskawa) b) discovery of tau lepton, made possible by high energy e+e- rings c) discovery of quarks and gluons, made possible by SLAC high energy electron linac d) discovery of resonances made possible by new energy realm, separated beams and large scale bubble chambers.

${ }^{4}$ In this document, the $\mathrm{ME}$ ' $\mathrm{NO} \nu \mathrm{A}$ ' beam will be referred to as the NuMI-NO $\nu \mathrm{A}$ beam. Other abbreviations: Charged Current (CC), Neutral Current (NC), Far Detector(FD), Near Detector(ND), Medium Energy(ME), Low Energy(LE), anti-neutrino mode Reverse Horn Current (RHC), neutrino mode Forward Horn Current (FHC) running. Estimates of event rates have been made assuming $6 \times 10^{20}$ P.O.T per year.
} 
MINOS 2010 data sample shown in black. Realistic estimates for the MINOS efficiency and energy resolution have been applied in the following studies.
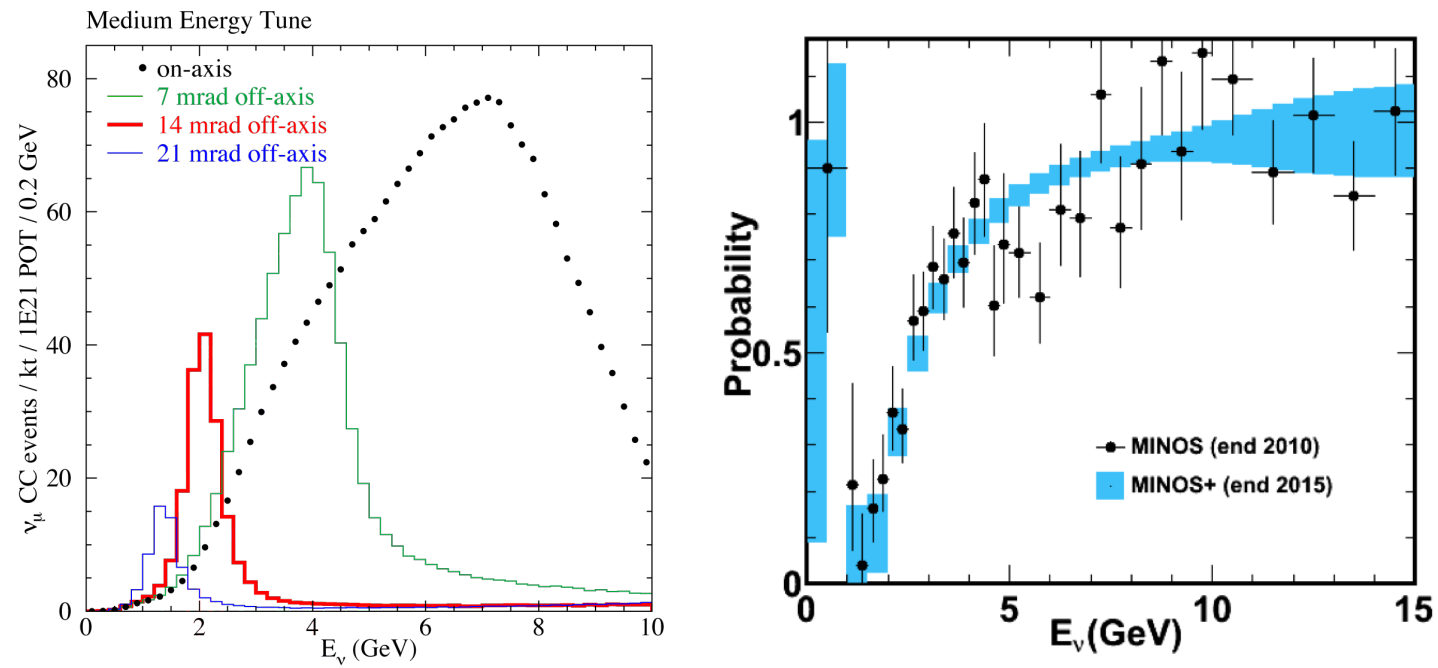

Figure 2: The energy spectra of neutrinos produced with the NUMI-NO $\nu \mathrm{A}$ beam (left). The black points show the spectrum of CC events which would be seen at MINOS, the red histogram is the spectrum of the events at the $\mathrm{NO} \nu \mathrm{A}$ experiment. The ratio of oscillated to un-oscillated rates is shown at right for MINOS+ with the present data points from the MINOS 5 year period up to 2010 overlaid in black.

\section{Physics reach of MINOS+}

What follows are the results of studies of the reach of the measurements which could be made in the NuMI-NO $\nu$ A beam. They are ordered in terms of probability of success, starting off with the measurements which are certain to contribute to world knowledge and ending with examples of much more speculative new physics which could be identified in the high energy neutrino energy spectrum. What is interesting is the very wide range of disparate new physics which could show up in this energy range :

- Measurement of $\sin ^{2} 2 \theta$ and $\Delta \mathrm{m}^{2}$ with higher precision

- Measurement of $\sin ^{2} 2 \bar{\theta}_{23}$ and $\Delta \overline{\mathrm{m}}^{2}$ with higher precision

- Study of High Energy Neutrinos

- Search for Sterile Neutrinos

- Search for Tau Neutrinos

- Non-Standard Interactions

- Measurement of the Neutrino Time of Flight 
- Search for Extra Dimensions

- Atmospheric Neutrinos

\subsection{Measurement of $\sin ^{2} 2 \theta$ and $\Delta \mathrm{m}^{2}$}

At energies between 4-10 GeV, MINOS+ will continue to improve the high precision measurement of $\Delta \mathrm{m}^{2}$ and $\sin ^{2} 2 \theta$ which can be combined with the results from $\mathrm{NO} \nu \mathrm{A}$ to provide accuracy towards $3 \%$ in $\sin ^{2} 2 \theta$ and $2 \%$ in $\Delta \mathrm{m}^{2}$. Figure 3 (left) shows the $90 \%$ parameter contours assuming a $50 \%$ fiducial mass for $\mathrm{NO} \nu \mathrm{A}$ during the first year of running. On the right are shown the same contours after three years of NuMI-NO $\nu \mathrm{A}$ running ( $\mathrm{NO} \nu \mathrm{A}$ construction is completed 6 months into year 2). For the contours shown below, a $4 \%$ energy resolution has been used for $\mathrm{NO} \nu \mathrm{A}$ where only quasi elastic events have been used. No background has been included for either MINOS+ or NO $\nu \mathrm{A}$. The simulations have assumed that at the start of running, full beam would be available (700kW), that $\mathrm{NO} \nu \mathrm{A}$ would be $50 \%$ complete on day 1 and fully complete on day 365 . It is possible that MINOS+ will have results with a very fast turnaround compared to $\mathrm{NO} \nu \mathrm{A}$ who will be carrying out their first analysis. The NC background constitutes the largest systematic error on the $\sin ^{2} 2 \theta$ measurement in MINOS. This will be improved with the higher energy of NuMI-NO $\nu \mathrm{A}$ beam where the NC cross-section is better known, and with the aid of improved measurements of secondary pion yields in neutrino interactions. While the improvement over the $\mathrm{NO} \nu \mathrm{A}$ only measurement after three years is small, in the first year or two it will be substantial. Furthermore, MINOS+ will provide an additional in-situ constraint on the $\nu_{\mu}$ contamination in $\bar{\nu}_{\mu}$ running for $\mathrm{NO} \nu \mathrm{A}$ which is a substantial concern when searching for $\delta_{C P}$.
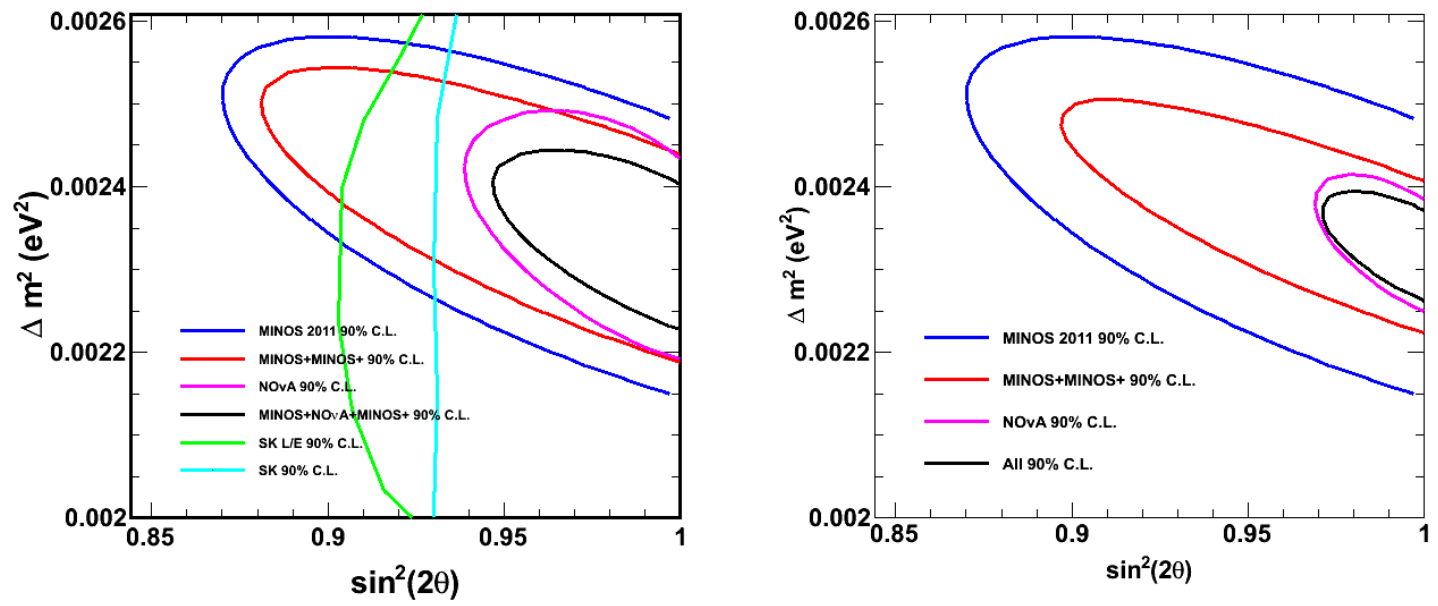

Figure 3: Contours showing the reach of MINOS in the measurement of the standard parameters $\Delta \mathrm{m}^{2}$ and $\sin ^{2} 2 \theta$. Left is after one year of MINOS which gets 6e20 POT exposure and $\mathrm{NO} \nu \mathrm{A}$, which averages $89 \%$ of its full FD mass running, shown together with Super-K contours from [9]. While Super-K have presented improved limits, they have not been published. The black contours show the combined precision for $\mathrm{NO} \nu \mathrm{A}$ and MINOS. Right shows the contours after 3 years of neutrino running. 

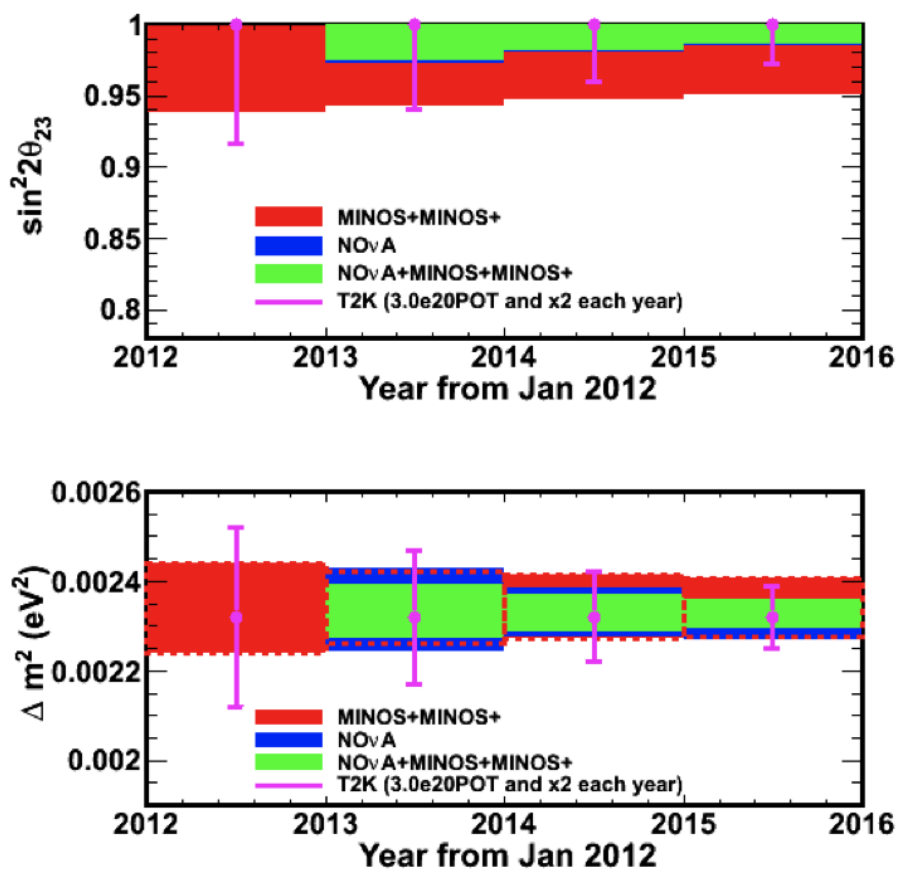

Figure 4: Estimated progression of the parameters $\Delta \mathrm{m}^{2}$ and $\sin ^{2} 2 \theta$. The T2K numbers are based on present sensitivity with $3 \mathrm{e} 19$ POT and an estimated 3e20 POT collected by start of MINOS+ running.

\subsection{Measurement of $\sin ^{2} 2 \bar{\theta}_{23}$ and $\Delta \overline{\mathrm{m}}^{2}$}

At energies between 4-10 GeV, MINOS+ can also continue to improve the high precision measurement of $\Delta \overline{\mathrm{m}}^{2}$ and $\sin ^{2} 2 \bar{\theta}_{23}$ in a similar way to that described above. Figure 5 (left) shows the $90 \%$ parameter contours using the same assumptions as above but assuming RHC (anti-neutrino) running instead of FHC (neutrino) running. The simulated value of $\sin ^{2} 2 \bar{\theta}_{23}$ and $\Delta \overline{\mathrm{m}}^{2}$ are those measured by MINOS [8]. 


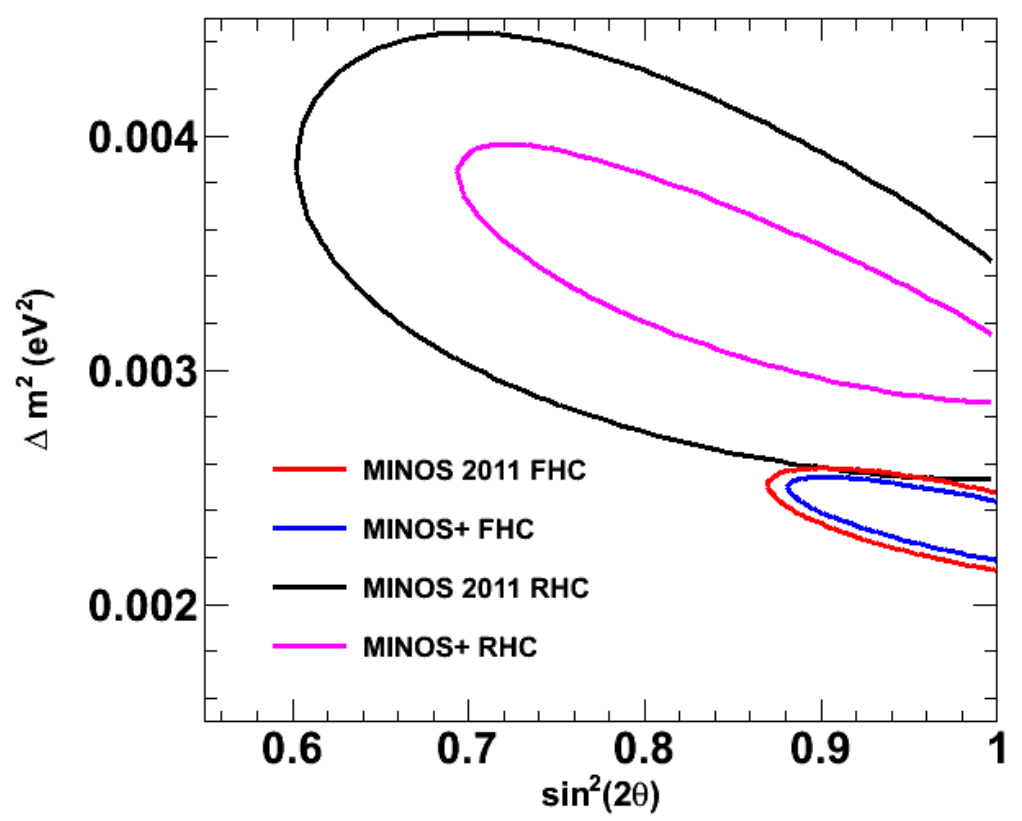

Figure 5: Contours showing the reach of MINOS+ in the measurement of the standard parameters $\Delta \overline{\mathrm{m}}^{2}$ and $\sin ^{2} 2 \bar{\theta}_{23}$ after one year of MINOS+ assuming 6e20 POT RHC (antineutrino) exposure. Also shown is the neutrino contour after one year of FHC (neutrino) running. Central values used are $\left|\Delta m_{32}^{2}\right|=\left(2.32_{-0.08}^{+0.12}\right) \times$ $10^{-3} \mathrm{eV}^{2}$ and $\sin ^{2}\left(2 \theta_{23}\right)>0.90$ (90\% C.L.)and $\Delta \overline{\mathrm{m}}^{2}=3.36_{-0.40}^{+0.46} \mathrm{eV}^{2}$ and $\sin ^{2} 2 \bar{\theta}_{23}=0.86 \pm_{-0.12}^{+0.11}$ (90\% C.L.). The MINOS 2011 results are the latest published results to date and do not include extra data taken but not yet analysed. 


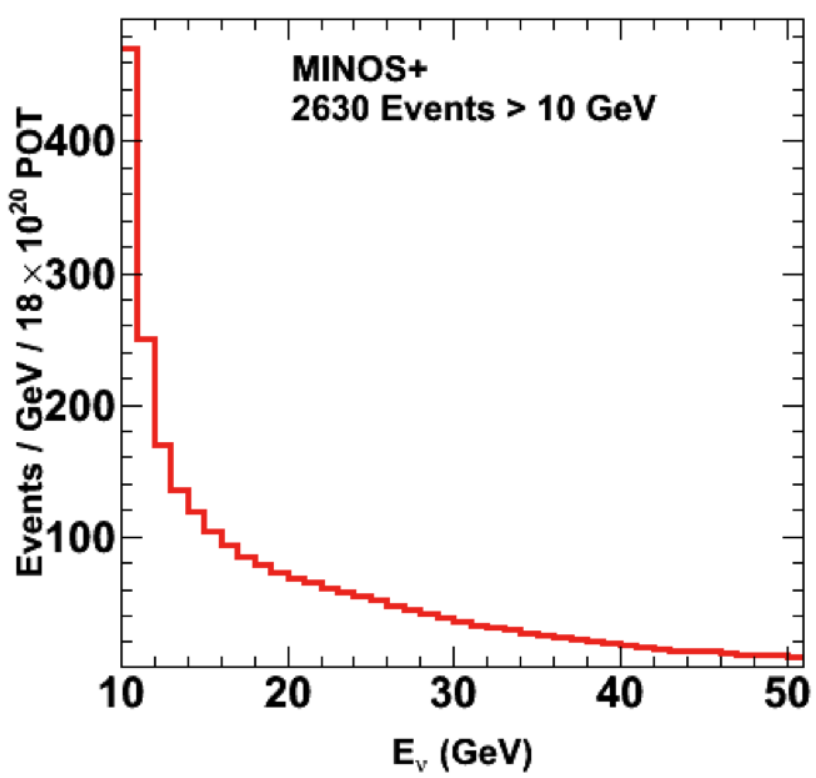

Figure 6: The yield of neutrinos above $10 \mathrm{GeV}$ in the NuMI-NO $\nu \mathrm{A}$ beam. The high energy part of the spectrum is another handle on non-standard behavior as it covers a range from L/E of 75 to 15 .

\subsection{High Energy Neutrinos}

The neutrinos in the high energy tail chiefly come from on-axis pions and kaons passing through the horns unscathed by focussing. Given $\Delta m_{23}^{2} \simeq \Delta m_{13}^{2} \simeq 2.3 \times 10^{-3} \mathrm{eV}^{2}$ and the flight length of $735 \mathrm{~km}$, the energy region $10 \leq E_{\nu} \leq 50 \mathrm{GeV}(\mathrm{L} / \mathrm{E}$ of $15-75 \mathrm{~km} / \mathrm{GeV}$ )

will not generate any oscillation in the traditional (PMNS) scenario. The largely unexplored high energy region offers a unique opportunity to search for physics beyond the PMNS oscillation. MINOS+ will provide the highest existing statistics $\nu_{\mu}$ and $\bar{\nu}_{\mu} \mathrm{CC}$ sample between $10 \leq E_{\nu} \leq$ $50 \mathrm{GeV}$. Of course, it should be noted that L/E is not necessarily the only figure of merit; new effects might have a different dependence. There will be a corresponding large data set available in the NC sample $\left(E_{H a d} \geq 5 \mathrm{GeV}\right)$. The ND will provide the un-oscillated neutrino spectrum in the high energy region with systematic precision better than the statistics. The spectrum from a three year data taking period is shown in Fig 6.

\subsection{Search for Sterile Neutrinos}

A possible solution to the MiniBooNE [13], LSND [14] and reactor antineutrino [15] anomalies is the existence of a fourth, sterile neutrino flavour $\nu_{\mathrm{s}}$, coupled to a fourth neutrino mass state $\nu_{4}$. The mass of this fourth state would need to be such that $m_{4}^{2}-m_{3}^{2}=\mathcal{O}\left(1 \mathrm{eV}^{2}\right)$. Such a sterile neutrino would cause the disappearance at high energies, in the MINOS Far Detector (FD), of muon neutrinos or antineutrinos produced by the NuMI beam. The NO $\nu$ A-era NuMI beam produces a large flux of high-energy $\nu_{\mu}$ or $\bar{\nu}_{\mu}$ at the MINOS FD. Therefore the MINOS+ experiment is well set up to search for such a sterile neutrino. 


\subsubsection{A fourth neutrino state}

The addition of a fourth neutrino mass and flavour state pair requires the PMNS mixing matrix to be generalized to four dimensions:

$$
U=\left(\begin{array}{cccc}
U_{e 1} & U_{e 2} & U_{e 3} & U_{e 4} \\
U_{\mu 1} & U_{\mu 2} & U_{\mu 3} & U_{\mu 4} \\
U_{\tau 1} & U_{\tau 2} & U_{\tau 3} & U_{\tau 4} \\
U_{s 1} & U_{s 2} & U_{s 3} & U_{s 4}
\end{array}\right)
$$

Using the standard formulism discussed in [3],

$$
\begin{aligned}
P\left(\nu_{\alpha} \rightarrow \nu_{\beta}\right)= & \delta_{\alpha \beta}-4 \sum_{j>i} \mathcal{R}\left(U_{\alpha j}^{*} U_{\beta j} U_{\alpha i} U_{\beta i}^{*}\right) \sin ^{2} \Delta_{j i} \\
& +2 \sum_{j>i} \mathcal{I}\left(U_{\alpha j}^{*} U_{\beta j} U_{\alpha i} U_{\beta i}^{*}\right) \sin \left(2 \Delta_{j i}\right),
\end{aligned}
$$

where $\Delta_{j i}=\left(m_{j}^{2}-m_{i}^{2}\right) L /(4 E)$ (with $L$ and $E$ the distanced traveled by and the energy of the neutrino respectively).

With the MINOS+ baseline and energies, oscillations driven by the mass splitting $\Delta m_{21}^{2}$ can be neglected; hence the approximation $\sin ^{2} \Delta_{21}=\sin \left(2 \Delta_{21}\right)=0$ is valid. This leads to the equivalent approximations $\Delta_{42}=\Delta_{41}$ and $\Delta_{32}=\Delta_{31}$, giving the following expression for the $\nu_{\mu}\left(\right.$ or $\left.\bar{\nu}_{\mu}\right)$ survival probability:

$$
\begin{aligned}
P_{\nu_{\mu} \rightarrow \nu_{\mu}}=1- & 4\left\{\left|U_{\mu 3}\right|^{2}\left(1-\left|U_{\mu 3}\right|^{2}-\left|U_{\mu 4}\right|^{2}\right) \sin ^{2} \Delta_{31}\right. \\
& +\left|U_{\mu 4}\right|^{2}\left|U_{\mu 3}\right|^{2} \sin ^{2} \Delta_{43} \\
& \left.+\left|U_{\mu 4}\right|^{2}\left(1-\left|U_{\mu 3}\right|^{2}-\left|U_{\mu 4}\right|^{2}\right) \sin ^{2} \Delta_{41}\right\}
\end{aligned}
$$

where

$$
\begin{aligned}
& U_{\mu 3}=-\sin \theta_{14} \sin \theta_{13} e^{-i \delta_{1}} \sin \theta_{24} e^{-i \delta_{2}}+\cos \theta_{13} \sin \theta_{23} \cos \theta_{24}, \\
& U_{\mu 4}=\cos \theta_{14} \sin \theta_{24} e^{-i \delta_{2}} .
\end{aligned}
$$

The angle $\theta_{13}$ is known to be small [11], so the approximation $\theta_{13}=0$ is made, which also makes the value of $\delta_{1}$ irrelevant. Measurements by short baseline reactor experiments suggest that $\theta_{14}$ is small (see appendix A.1); even if the signal suggested by the reactor antineutrino anomaly is positive, this only suggests a small value for $\theta_{14}$. Hence the approximation $\sin \theta_{24} \cos \theta_{14} \approx \sin \theta_{24}$ is also made. The matrix elements then simplify to

$$
U_{\mu 3}=\sin \theta_{23} \cos \theta_{24}, \quad U_{\mu 4}=\sin \theta_{24} e^{-i \delta_{2}}
$$

For this study, it is assumed that $m_{4} \gg m_{3}$. Hence, within the resolution of the MINOS detectors, $\sin ^{2} \Delta_{41}$ and $\sin ^{2} \Delta_{43}$ average to $\frac{1}{2}$. Therefore

$$
\begin{aligned}
P_{\nu_{\mu} \rightarrow \nu_{\mu}} & =1-4\left\{\left|U_{\mu 3}\right|^{2}\left(1-\left|U_{\mu 3}\right|^{2}-\left|U_{\mu 4}\right|^{2}\right) \sin ^{2} \Delta_{31}+\frac{\left|U_{\mu 4}\right|^{2}}{2}\left(1-\left|U_{\mu 4}\right|^{2}\right)\right\} \\
& =1-\sin ^{2}\left(2 \theta_{23}\right) \cos ^{4} \theta_{24} \sin ^{2} \Delta_{31}-\frac{1}{2} \sin ^{2} 2 \theta_{24} .
\end{aligned}
$$




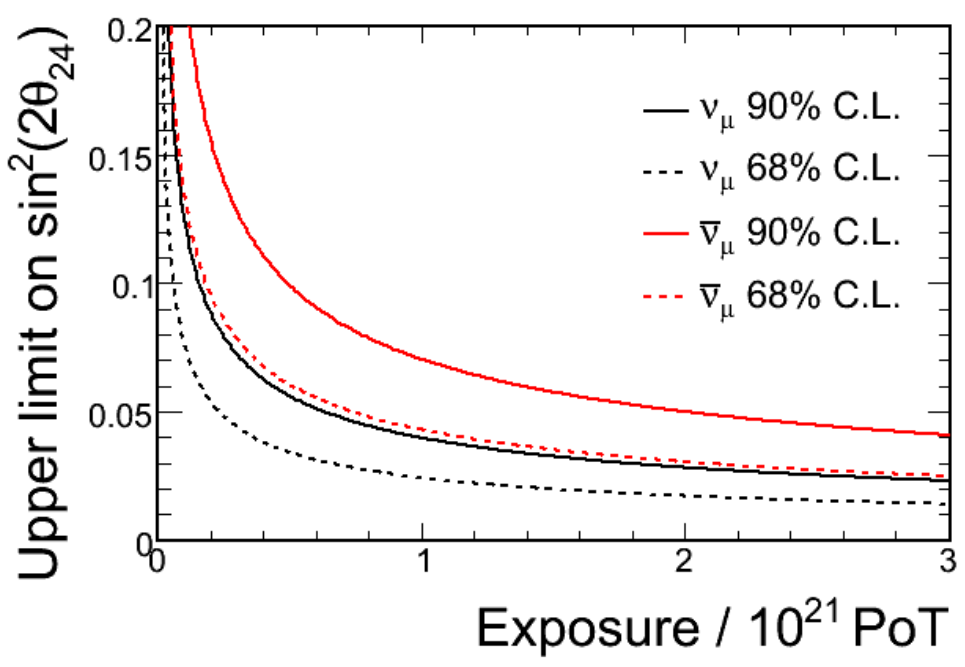

Figure 7: The sensitivity of the MINOS+ to the mixing angle $\theta_{24}$, as a function of exposure in protons on target for both neutrino and antineutrino running.

(Note that the parameter $\delta_{2}$ does not appear in the expression for the disappearance probability.)

The existence of a sterile neutrino will also cause the depletion of neutral current (NC) events in the MINOS FD, since a sterile neutrino does not interact through the neutral current. The probability of oscillation into a sterile neutrino is given by

$$
\begin{aligned}
P\left(\nu_{\mu} \rightarrow \nu_{\mathrm{s}}\right)= & 4 \mathcal{R}\left\{\left|U_{\mu 3}\right|^{2}\left|U_{s 3}\right|^{2} \sin \Delta_{31}+\left|U_{\mu 4}\right|^{2}\left|U_{s 4}\right|^{2} \sin ^{2} \Delta_{41}\right. \\
& \left.+U_{\mu 4}^{*} U_{s 4} U_{\mu 3} U_{s 3}^{*}\left(\sin ^{2} \Delta_{31}-\sin ^{2} \Delta_{43}+\sin ^{2} \Delta_{41}\right)\right\} \\
& +2 \mathcal{I}\left\{U_{\mu 4}^{*} U_{s 4} U_{\mu 3} U_{s 3}^{*}\left(\sin 2 \Delta_{31}-\sin 2 \Delta_{41}+\sin 2 \Delta_{43}\right)\right\} .
\end{aligned}
$$

The relevant elements of the PMNS matrix are given by

$$
\begin{aligned}
U_{\mu 3}= & -\sin \theta_{14} \sin \theta_{13} e^{-i \delta_{1}} \sin \theta_{24} e^{-i \delta_{2}}+\cos \theta_{13} \sin \theta_{23} \cos \theta_{24}, \\
U_{\mu 4}= & \cos \theta_{14} \sin \theta_{24} e^{-i \delta_{2}}, \\
U_{s 3}= & -\sin \theta_{14} \cos \theta_{24} \cos \theta_{34} \sin \theta_{13} e^{-i \delta_{1}} \\
& -\cos \theta_{13} \sin \theta_{23} \cos \theta_{34} \sin \theta_{24} e^{i \delta_{2}}-\cos \theta_{13} \cos \theta_{23} \sin \theta_{34}, \\
U_{s 4}= & \cos \theta_{14} \cos \theta_{24} \cos \theta_{34} .
\end{aligned}
$$

Hence the NC disappearance probability is sensitive to the angle $\theta_{34}$ (primarily through the $\sin \theta_{34}$ term in $\left.U_{s 3}\right)$ as well as to $\theta_{24}$. The $\theta_{14}$ dependence is minimal, since it only features through $\cos \theta_{14}$ which, for small $\theta_{14}$, is approximately unity.

\subsubsection{MINOS+ sensitivity to sterile neutrinos}

Figure 7 shows the MINOS sensitivity to $\sin ^{2}\left(2 \theta_{24}\right)$ as a function of NuMI-NO $\nu$ A beam exposure, for both neutrino and antineutrino running. Here, as throughout this study, the values $\Delta m_{32}^{2}=$ $2.32 \times 10^{-3} \mathrm{eV}^{2}$ and $\sin ^{2}\left(2 \theta_{23}\right)=1.0$ are used.

MINOS+ is sensitive to sterile neutrinos in a way that is highly complimentary to other searches. Reactor experiments [15], which measure at $\nu_{\mathrm{e}}$ disappearance, are sensitive only to the mixing angle 
$\theta_{14}$ (see appendix A.1). The LSND and MiniBooNE measurements that search for $\nu_{\mathrm{e}}$ appearance in a $\nu_{\mu}$ beam are sensitive to a combination of the angles $\theta_{14}$ and $\theta_{24}$ (see appendix B). The MINOS+ experiment, which looks for $\nu_{\mu}$ (or $\bar{\nu}_{\mu}$ ) disappearance, makes a very clean measurement of $\theta_{24}$, with little sensitivity to $\theta_{14}$ (as shown above).

The MINOS sensitivity to $\theta_{24}$ after two years of running with neutrinos or antineutrinos is shown in Figure 8. This is compared to the existing limits: $\nu_{\mu}$ and $\bar{\nu}_{\mu}$ disappearance searches by MiniBooNE, CDHS and CCFR. Figure 9 shows the effect on the MINOS+ ND and FD energy spectra of oscillations including a $\Delta m_{42}^{2}$ of $1.5 \mathrm{eV}^{2}$ and $\sin ^{2}\left(2 \theta_{24}\right)=0.5$ (approximately the MiniBooNE $90 \% \bar{\nu}_{\mu}$ limit at this mass splitting; see figure 9). Figure 9 shows that over the range of mass splittings probed, MINOS+ can set significantly stronger limits on the angle $\theta_{24}$ than any previous experiment. 


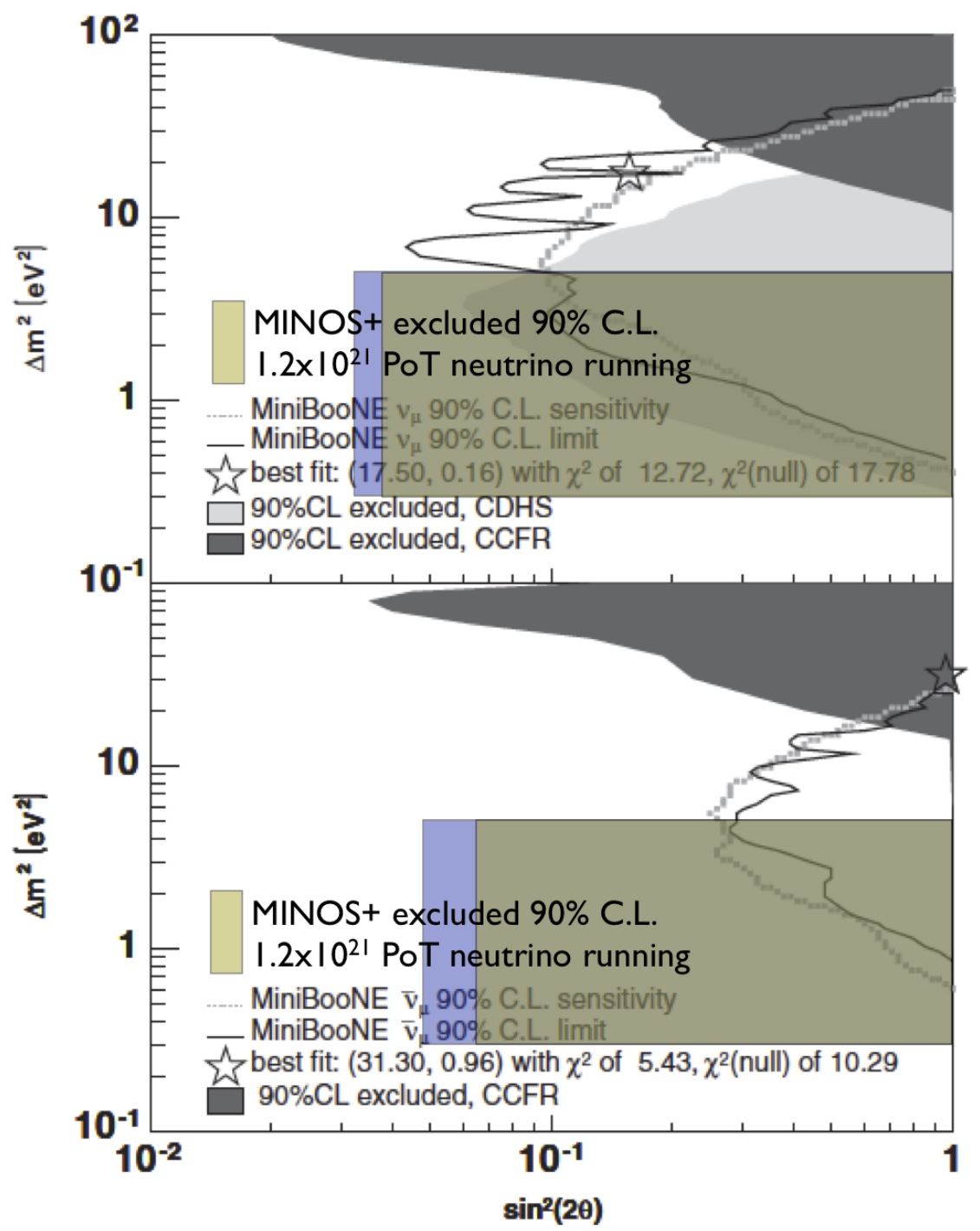

Figure 8: The sensitivity of MINOS+ to the mixing angle $\theta_{24}$, after two years of running in neutrino (top) or antineutrino (bottom) mode. The green region is that excluded by $\nu_{\mu}$ disappearance whereas the blue region is further excluded by the NC disappearance search. Also shown are the comparable limits set by other experiments which search for $\nu_{\mu}$ disappearance. 

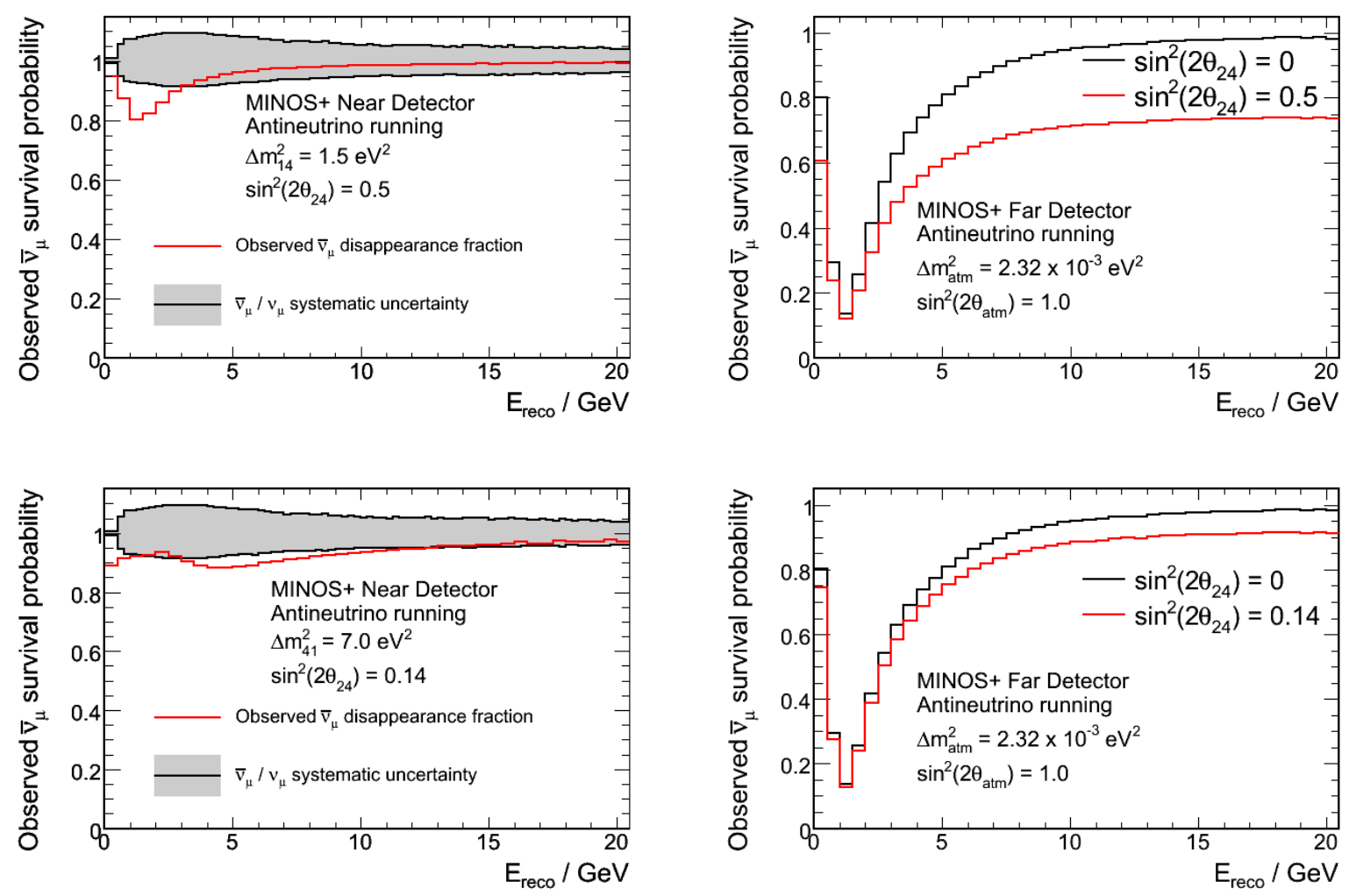

Figure 9: The effect of oscillations in the MINOS+ ND (left) and FD (right), including the effect of a fourth, sterile neutrino flavour coupled to a high-mass state in the anti-neutrino reactor anomaly allowed range ( two examples, $\Delta \mathrm{m}^{2}=1.5 \mathrm{eV}^{2}$ and $\Delta \mathrm{m}^{2}=7.0 \mathrm{eV}^{2}$ ) with a $\sin ^{2} 2 \theta_{24}$ of 0.5 (upper) and 0.14 (lower). The former $\sin ^{2} 2 \theta_{24}$ value is used as this represents the best previous direct limit on this parameter for antineutrinos from MiniBooNE. The error band shown in the case of the ND are the beam and cross section uncertainties on the ratio of antineutrino to neutrino energy spectra. 
The recent MINOS search for sterile neutrinos [3] uses the NC event sample because all active flavors participate in the neutral-current interaction, so there should be no difference between the observed and expected number of $\mathrm{NC}$ events at the far detector. If mixing occurs between active and sterile flavors, then the observed NC event sample will show an energy dependent deficit of events compared to the expectation. However, the $\mathrm{NC}$ events can be combined with a precise measurement of $\nu_{\mu} \mathrm{CC}$ disappearance in the NuMI-NO $\nu \mathrm{A}$ beam to push down further on the limits on sterile neutrino flavor. The $\mathrm{NC}$ disappearance measurement is actually sensitive to a mixture of $\theta_{24}$ and $\theta_{34}$ as the neutrinos which oscillate into taus also disappear below tau threshold. This obviously has an energy dependence as the tau appearance probability increases with energy. The mathematics is discussed in Appendix C.

\subsection{Search for Tau Neutrinos}

Figure 10 (upper) shows the spectra for NC events and tau events expected for one year of MINOS+ running. The tau lepton threshold in neutrino events is $3.6 \mathrm{GeV}$, but the production cross section rises slowly. In one year $\left(6 \times 10^{20} \mathrm{POT}\right)$ of NUMI-NO $\nu \mathrm{A}$ beam neutrino running, $1636 \mathrm{NC}$-induced events are expected in the MINOS fiducial volume and with $\Delta \mathrm{m}^{2}=2.32 \times 10^{-3} \mathrm{eV}^{2}$ and $\sin ^{2} 2 \theta=$ 1.0, $87 \nu_{\tau}$ events are expected. While the number of $\nu_{\tau}$ events is small compared to the NC background, there are certain kinematical cuts which can be made to enhance either the hadronic decay of taus over the NC background or the muonic decay over CC background. Such studies are in their infancy, but Figure 10 (lower) shows the number of NC events compared to tau events plotted as a function of the individual measured $p_{T}$ of each hadron as a function of energy cut. These are for demonstration purposes only, and are not normalized to realistic numbers of events.

\subsection{Non-Standard Interactions}

The non-standard interactions of Kopp, Machado and Parke [18] predict differences between oscillation rates for neutrinos and anti-neutrinos. Figure 11 shows these predictions for different values of the model parameters at left. There are noticeable differences between neutrinos and antineutrinos at higher energies, but also differences between low and high energy predictions for neutrinos and anti-neutrinos separately. The sensitivity to this has been studied using a dataset comprising the $7.1 \times 10^{20}$ POT FHC (neutrino) running, the $1.7 \times 10^{20}$ POT RHC (anti-neutrino) running and an additional $1.7 \times 10^{20}$ POT RHC MC dataset which has been generated using the $\nu_{\mu} \mathrm{CC}$ oscillation parameters. This dataset will produce the strongest limit on the NSI parameters that MINOS could achieve in the LE configuration. The contour is shown in Figure 12.

The fractional difference in event rate between neutrinos and antineutrinos integrated over the 5-10 GeV region as a function of NSI parameter $\epsilon_{\mu \tau}$ is shown at right. A full explanation of this parameter is given in Appendix D. After two years, MINOS would have an accuracy on this integrated value of about 1\% (statistical error on 6000 events), which would correspond to a limit on the $\epsilon_{\mu \tau}$ of approximately -0.07 after 1 year and -0.04 after two years. This is compared to the limit imposed by the NSI contour (Figure 12) of $\epsilon_{\mu \tau}>0.26$, shown in Figure 13, and clearly extends the reach of this study. ${ }^{5}$ See Appendix D for a fuller explanation of the NSI parameters used.

\footnotetext{
${ }^{5} \epsilon_{\mu \tau}$ is a parameter where the sign of the physics comes from the sign of the matter potential, $\mathrm{V}$, so in the study carried out for MINOS it is chosen to be positive (and real, where in general it is complex). Parke's equation (12) says that the survival probability is invariant under multiplying by -1 , so it is equivalent
} 

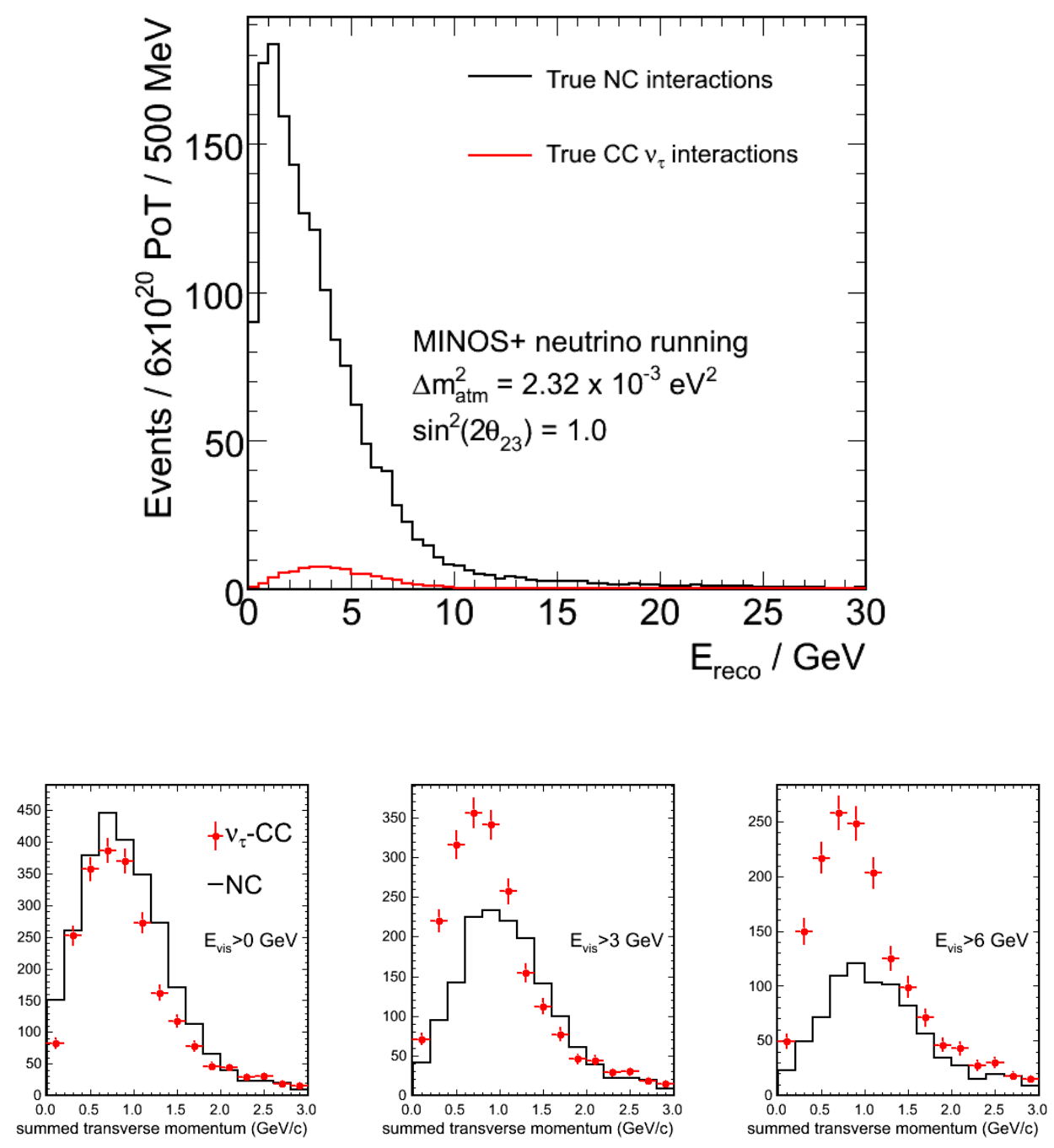

Figure 10: The expected NC event spectrum and the expected number of tau events produced in the NuMI$\mathrm{NO} \nu \mathrm{A}$ beam (upper) and the $\mathrm{p}_{T}$ distribution of $\mathrm{NC}$ and tau events as a function of energy cut (lower). 


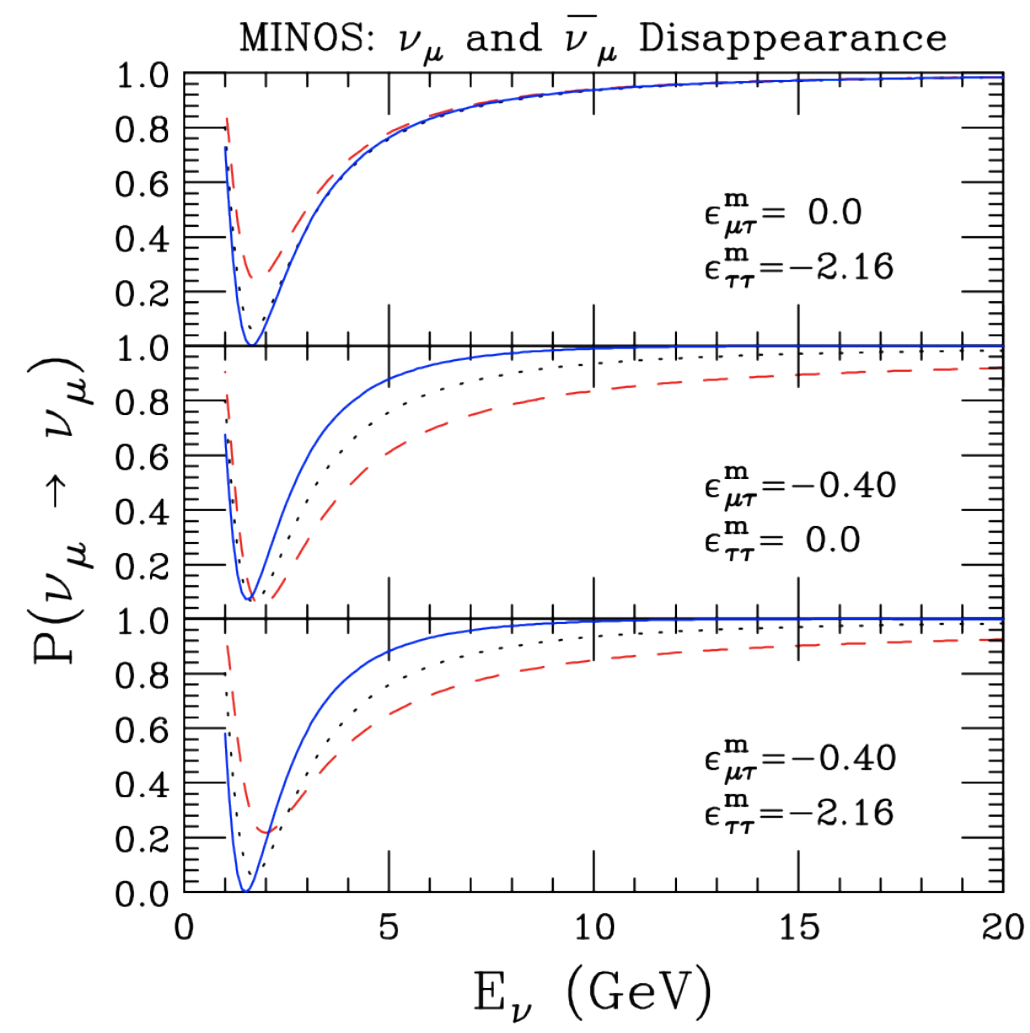

Figure 11: Predictions of the Kopp, Machado and Parke non-standard interaction probability for different values of the model parameters. The blue lines are the NSI expectation for neutrinos, the red dashed lines are anti-neutrinos and the black dotted line is the standard expectation for no NSI.

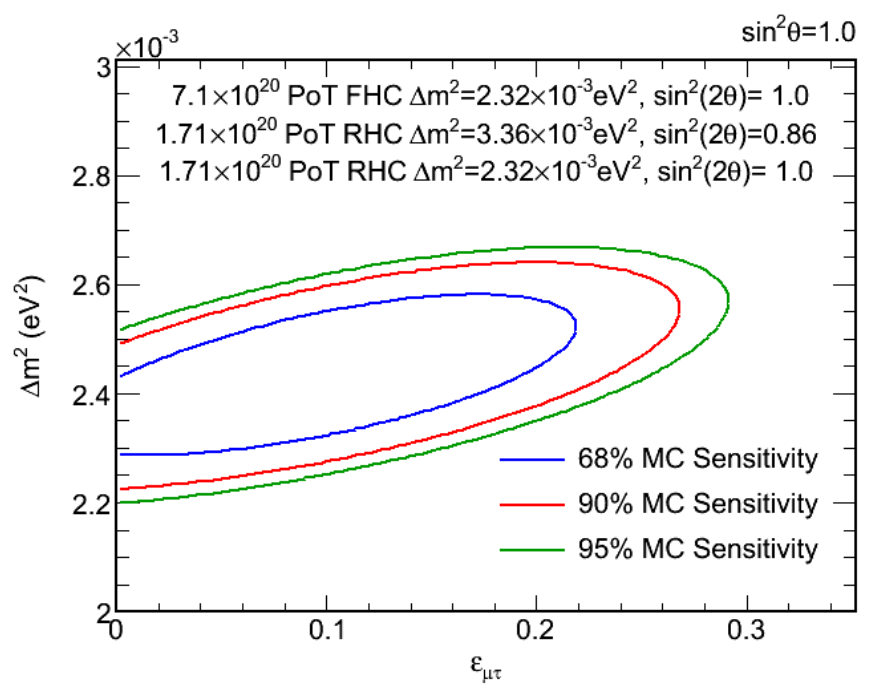

Figure 12: The sensitivity to $\epsilon_{\mu \tau}$ with $3.5 \mathrm{e} 20$ POT of RHC (see text) and 7.1e20 POT FHC running. 


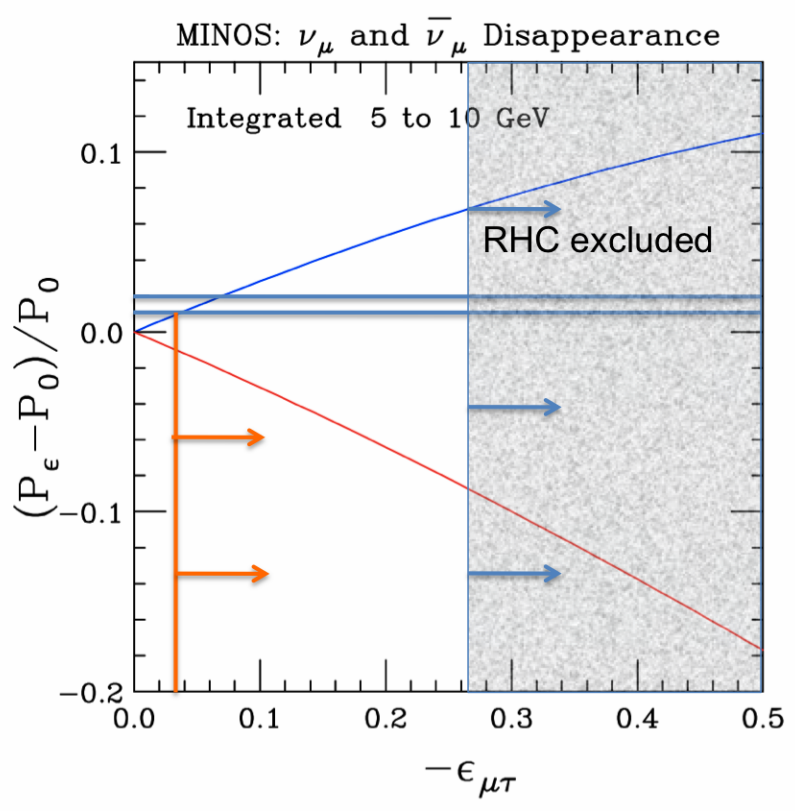

Figure 13: Predictions of the Kopp, Machado and Parke fractional non-standard interaction probability integrated over the $5-10 \mathrm{GeV}$ region as a function of $\epsilon_{\mu \tau}$ for neutrinos (blue) and anti-neutrinos (red), showing the most stringent exclusion zone expected from the MINOS FHC and RHC LE running at $\epsilon_{\mu \tau}>0.26$ (blue vertical line) and that expected from 2 years of neutrino running in MINOS+ (orange vertical line). 


\subsection{Measurement of the $\nu_{\mu}$ time of flight}

The best limit on the speed of $e$-type neutrinos comes exclusively from the observation of neutrinos from SN1987A which makes specific assumptions about the supernova data to show that $\left|v_{\nu} / c-1\right|<$ $2 \times 10^{-9}[25]$ [26]. One of the neutrino beams constructed thirty years ago for the debut of Fermilab was the site of the first and best lab based measurement of the speed of a neutrino [23]. The experiment showed for $\mu$-type neutrinos that $\left|v_{\nu} / c-1\right|<40 \times 10^{-6}$ [24]. The MINOS experiment achieved $\left|v_{\nu} / c-1\right|<5.1 \pm 2.9 \times 10^{-5}[16]$, the precision of which was dominated by the systematic error of $64 \mathrm{~ns}$ from the existing electronics.

MINOS+ would provide an opportunity to make a more precise lab based measurement of the muon neutrino speed. With additional hardware to measure the propagation time to an accuracy of $\pm 1 \mathrm{~ns}$ and an improvement in the accuracy of the $735.34 \mathrm{~km}$ distance to $\pm 0.1 \mathrm{~m}$ the $\nu_{\mu}$ speed can be ascertained at the level of $\left|v_{\nu} / c-1\right|<5 \times 10^{-7}$. This measurement could not make an impact on the absolute neutrino mass, the accuracy of this would be at best about $2 \mathrm{MeV}$, but the neutrino speed is an independent observable, and as such has not been measured at the proposed accuracy without unknowns which must be estimated. Recently, several papers have proposed a limiting speed for neutrinos which is less than $c$ [27]. The time of flight measurement could also test for Lorentz invariance violation in neutrinos [28].

Presently, the baseline distance is known to only $0.7 \mathrm{~m}$ but a remeasurement of the distance between a benchmark near the mineshaft and one in the MINOS ND hall should improve this substantially. The bunching of the proton beam imposed by the accelerator RF is the essential feature which allows events at the two locations to be correlated. The structure consists of pulses of $2 \mathrm{~ns}$ width separated by 19 ns. The transit time to Soudan will be measured, modulo the 19 ns period of the beam microstructure by atomic clocks at each end of the journey and the time of each pulse train will be compared between the two clocks. A full description of the setup is attached to this proposal.

As proposed by Shrock [39] the voids between proton buckets offer the complementary opportunity of a search for weakly-interacting particles that are massive, in our case above about $10 \mathrm{MeV}$.

\subsection{Search for Extra Dimensions}

It is somewhat surprising to find that the MINOS data has already ruled out extra dimensions to a scale of about 1 micron! [12]. This is another very different example of how new physics could be found in the energy range accessible to MINOS+. Machado, Nunokawa and Zukanovich Funchal have produced the plot shown in Figure 14 (left) to demonstrate the effect an extra dimension at the level of $5 \times 10^{-7} \mathrm{~m}$ would have on the region of the neutrino spectrum available to MINOS+. It should be noted that this model requires the existence of the right-handed (and therefore sterile for these L/E) neutrino, which is a consequence of the neutrino having non-zero mass. Table top experiments devised to test for deviations of Newtonian gravity can only probe large extra dimensions of sub-millimeter sizes. The most stringent upper limit given by a torsion pendulum instrument is $200 \mu \mathrm{m}$ at $95 \%$ C.L. for the size of the largest flat dimension regardless of the number of extra dimensions [17]. At higher neutrino energy, the effects are larger and well within the reach of one year of data taking in the NUMI-NO $\nu \mathrm{A}$ beam. Figure 14 (right) gives the status of the world's search for extra dimensions by the same authors. 

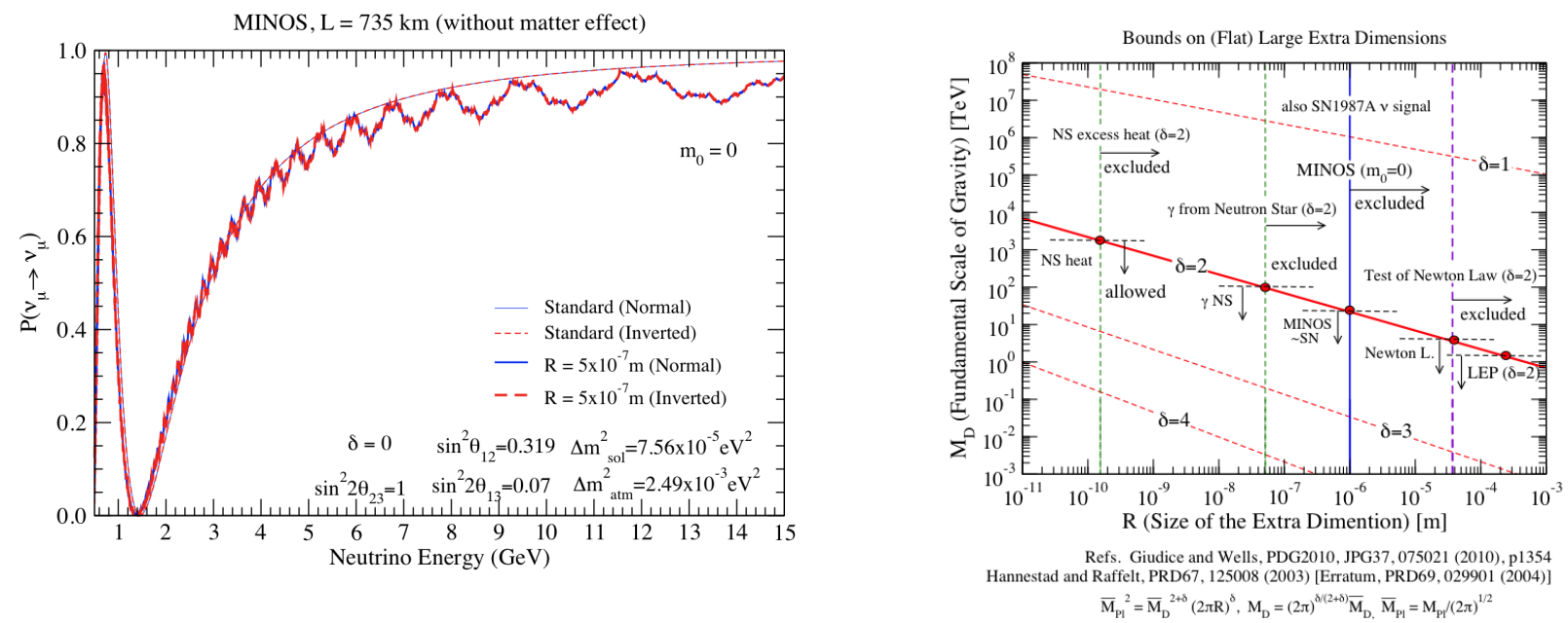

Figure 14: Predictions of the Machado, Nunokawa and Zukanovich Funchal model for extra dimensions using two years of MINOS running in the NuMI-NO $\nu \mathrm{A}$ beam (left) with $\mathrm{a}=510^{-7} \mathrm{~m}$ for both normal (blue line) and inverted (red line) hierarchy. and at right using present data comparing with other methods (right)

\subsection{Atmospheric Neutrinos}

The MINOS ability to separate the muon charge gives MINOS the ability to measure atmospheric neutrinos with oscillation parameters for neutrinos and anti-neutrinos separately, but with the same type of neutrinos as Super-K's. The measured contour for the present data and the sensitivity for data taken up to end of 2015 are shown in Figure 15.

Of great interest to the field is the neutrino mass hierarchy. One absolutely intrinsic characteristic of the neutrino, that of its fundamental nature, is as yet unknown. If in the next few years, the hierarchy can be shown to be the Inverted Hierarchy (IH) then the next generation of neutrino-less double beta decay experiments will either observe the process or be able to say that the neutrinos are of Dirac type. Figure 16 shows the $\chi^{2}$ difference between the hypotheses of the IH and the NH as a function of the energy versus zenith angle for atmospheric neutrinos (left) and anti-neutrinos (right). There is a region in both plots where a difference is apparent. This comes from two effects: 1) the sub-dominant oscillation of the muon neutrinos (and anti-neutrinos) into electron neutrinos (and anti-neutrinos) with the subsequent loss of rate in both muon neutrino and anti-neutrino rates as a function of $\mathrm{L} / \mathrm{E}$; and 2) the atmospheric electron neutrinos oscillating to muon neutrinos (which are then identified in the detector) as a function of $\mathrm{L} / \mathrm{E}$. This atmospheric monte carlo has been generated with a $\sin ^{2} \theta_{13}$ of 0.1 and normal hierarchy (for example), and is then compared with the prediction for the two different hypotheses. The $\chi^{2}$ bins from both plots are added to give one value of $\Delta \chi^{2}=\chi^{2}-\chi_{\min }^{2}$ for each generated value of $\sin ^{2} 2 \theta_{13}$ as shown in the bottom plot.

Super-K [30] presented a 1.6 sigma preference for the IH from measuring the difference in the number of electron neutrino events as a function of energy and distance coming from the subdominant muon to electron neutrino oscillation. If the MINOS 60 ktyr contour was combined with the Super-K contour, it would lead to a $>2 \sigma$ measurement of the mass hierarchy. These two measurements are very independent as they feature independent data sets, one looking at electron neutrino appearance, the other looking at muon neutrino appearance and disappearance. 

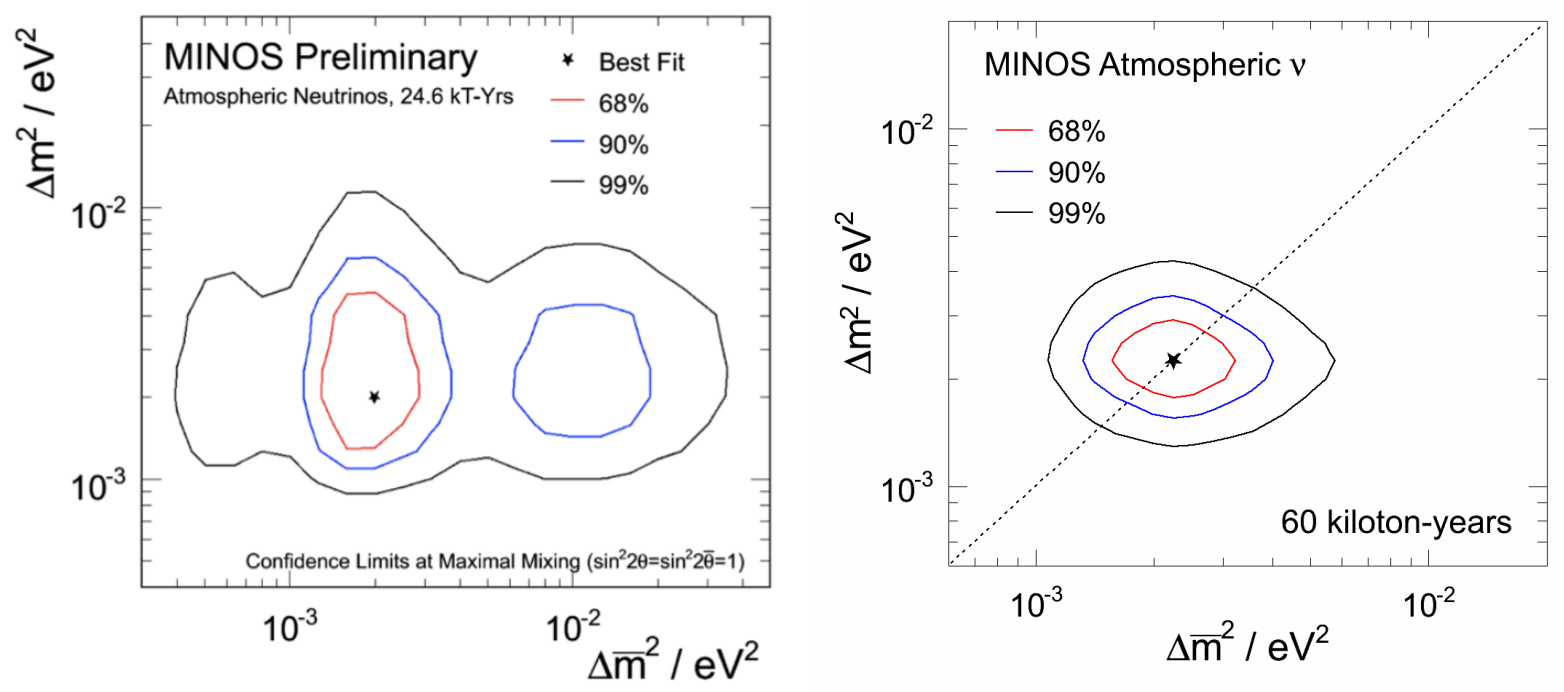

Figure 15: Plot of $\Delta \mathrm{m}^{2}$ versus $\Delta \overline{\mathrm{m}}^{2}$ for 27ktyrs (five years) of running (data) and 60 ktyrs of MINOS running (MC prediction). This latter corresponds to data taking until end of 2015, a three year run of MINOS+ 

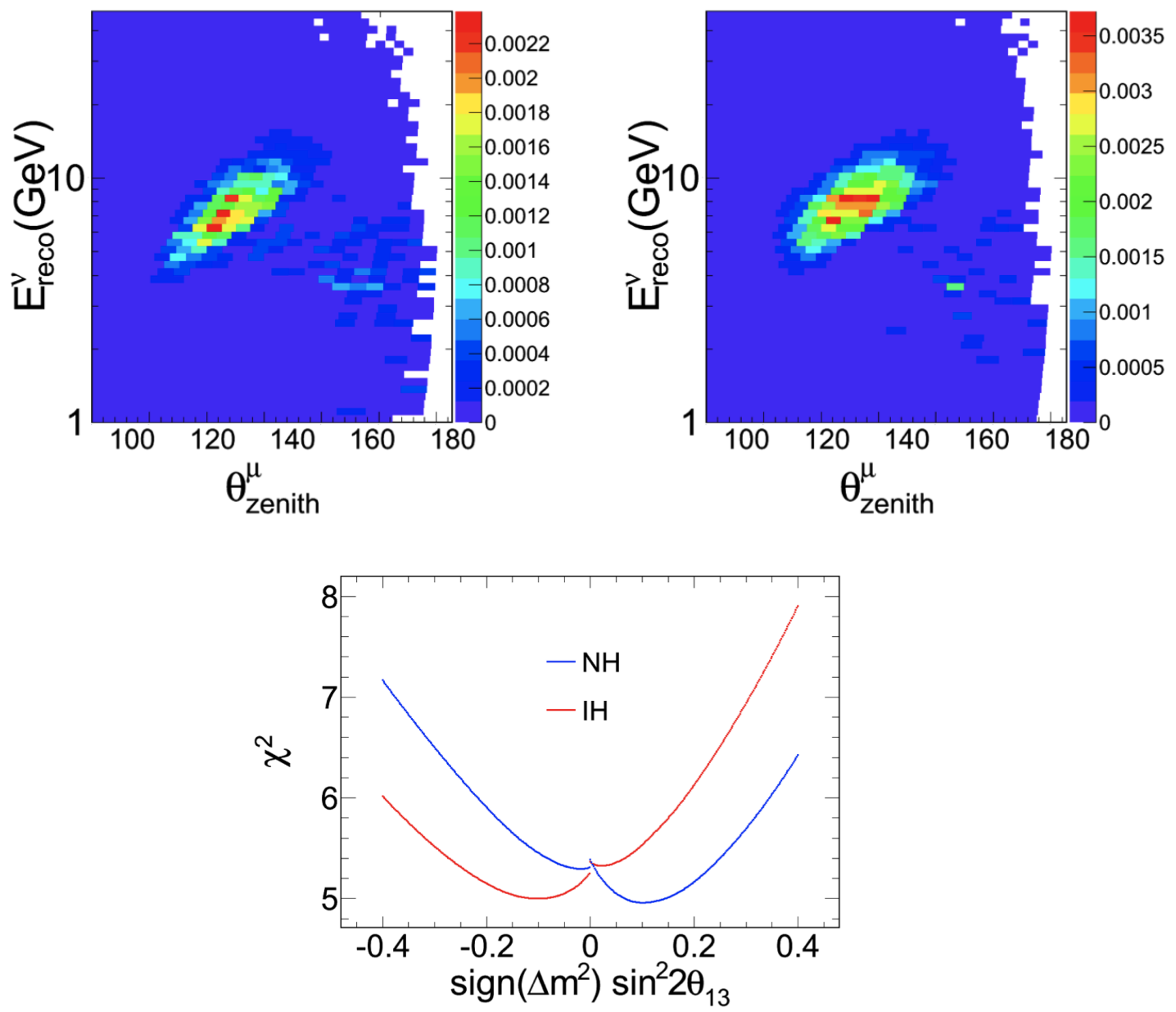

Figure 16: The plots at the top show the difference in the $\chi^{2}$ between $\mathrm{IH}$ and $\mathrm{NH}$ as a function of the energy versus zenith angle of atmospheric neutrinos (left, top) and anti-neutrinos (right, top). This atmospheric monte carlo has been generated with a $\sin ^{2} 2 \theta_{13}$ of 0.1 which leads to matter effects which differ between the two hierarchies. The sum of $\chi^{2}$ for both plots is shown in the bottom figure which can be used to read off the expected value of $\Delta \chi^{2}$ between IH and NH hypotheses of approximately 0.5 for $\sin ^{2} 2 \theta_{13}=0.15$ for an exposure of 60 ktyrs 

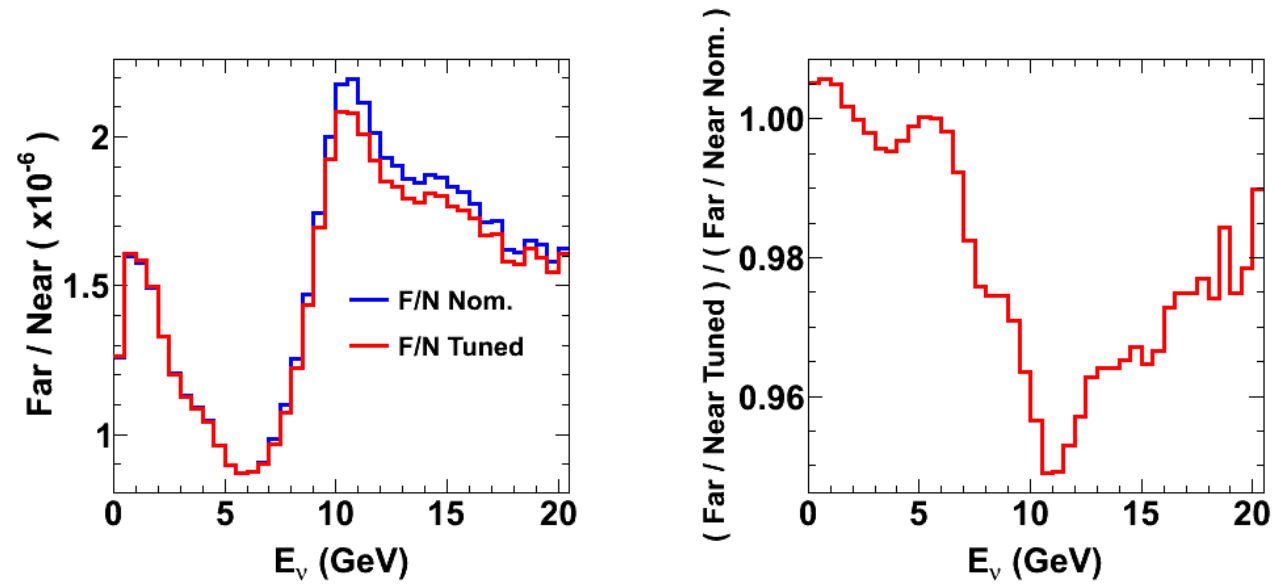

Figure 17: The F/N ratio is plotted as a function of energy for reweighted (red) and the nominal MC (blue) at left. Also shown is the ratio of the two, showing that the prediction is better than $5 \%$ per $0.5 \mathrm{GeV}$ bin below $10 \mathrm{GeV}$, at right.

\section{Systematic Uncertainties on the Far Detector Prediction}

MINOS has used a reweighting scheme to fit the generated MC flux to the data in order to reduce the initial disagreement between the ND data and MC (most notably the high energy tail found in the data which is $30 \%$ higher than the raw $\mathrm{MC}$ ). We can use this reweighting procedure as a conservative estimate for our systematic error on the prediction of the FD spectrum in the region of 4-10 GeV. This in turn can be used as a tool to study the estimated systematic error on the beam prediction at the FD. The difference in the prediction between using this reweighting correction and not using it is taken as a worst case systematic error. This is shown in Figure 17 where the far event rate divided by the near event rate $(\mathrm{F} / \mathrm{N}$ ratio) is plotted as a function of energy for reweighted (red) and the nominal MC (blue). Also shown is the ratio of the two, showing that the prediction is better than $5 \%$ per $0.5 \mathrm{GeV}$ bin below $10 \mathrm{GeV}$. This is a very conservative estimate of the size of the systematic error on the FD prediction. This is about the level of the statistical error after three years of running in the NuMI-NO $\nu \mathrm{A}$ beam. It is also worth noting that the existing dominant systematic uncertainties on energy scale and NC background (as already noted in sections 4.1 and 4.3) should decrease at the higher energies for which MINOS was originally designed [19].

\section{The Neutrino Beam Flux}

The off-axis $\mathrm{NO} \nu \mathrm{A}$ detectors along with the on-axis MINOS detectors and a measurement of the electron component of the beam from MINER $\nu$ A should provide enough information to substantially improve the MC flux models and through the use of fits to special runs, could, in principle, allow the flux to be known with unprecedented accuracy (for a neutrino beam).

MINOS has used beam matrices in the past for extrapolating the beam from the ND to the FD, but these plots (as shown in Figure 18) are a convenient way of seeing how the energy distributions are related in two detectors (in any position) in the same beam. The matrix element $\mathrm{M}_{i j}$ gives the probability that the distribution of secondary hadrons which produce $\nu_{\mu}$ 's of energy $\mathrm{E}_{i}$ in the ND 

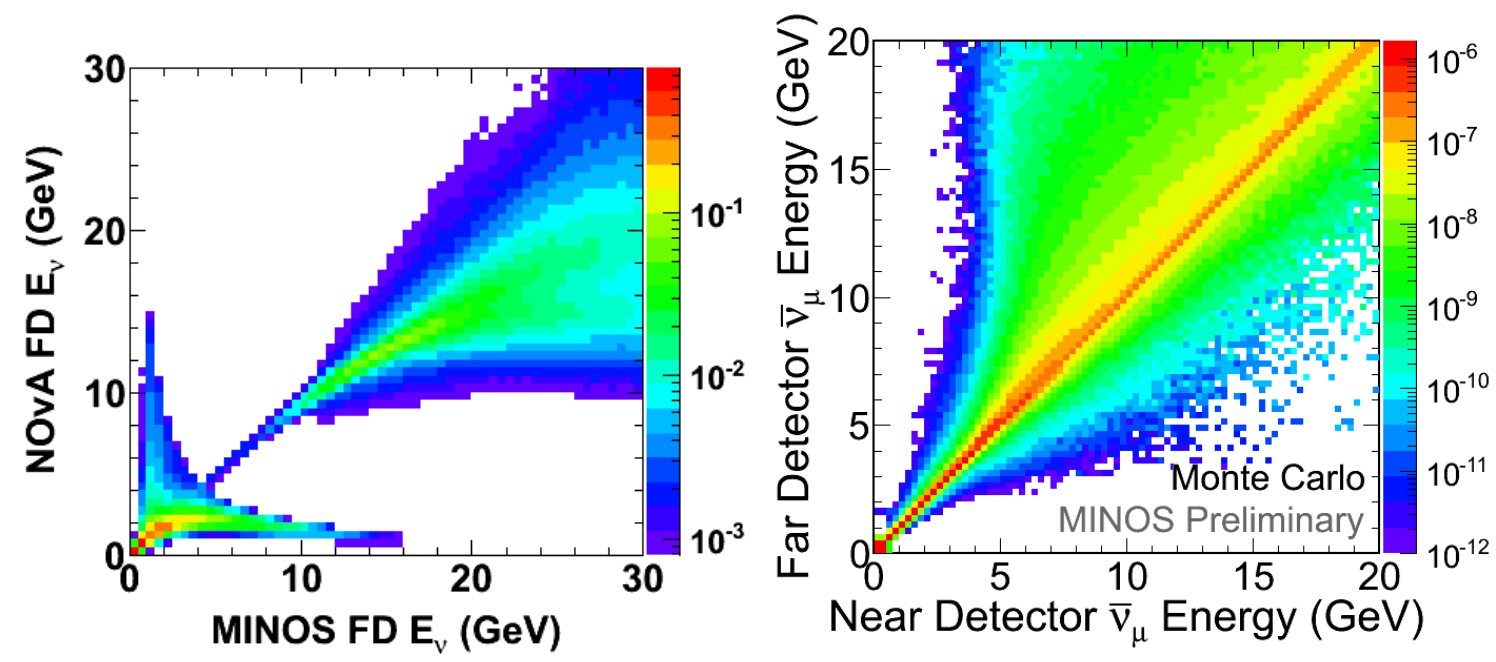

Figure 18: The beam matrix as calculated for the FHC MINOS ND and FD (right) and the off-axis $\mathrm{NO} \nu \mathrm{A}$ detector compared to the on-axis MINOS FD (left).

will give $\nu_{\mu}$ 's of energy $\mathrm{E}_{j}$ in the $\mathrm{FD}$ (or indeed in theNO $\nu \mathrm{A} \mathrm{ND}$ ). This probability is entered in the beam matrix histogram. The energy is slightly different in the two cases due to differences in acceptance of the two detectors. In MINOS+ there would be a third detector, the NO $\nu \mathrm{A}$ off-axis detector, which could also be included in the matrix. Measurements at the two near detectors could provide important constraints on the the neutrino flux. To give an idea of the kind of studies we could do, Figure 18 shows the two beam matrices relating the MINOS and NO $\nu \mathrm{A}$ ND fluxes (left) and the MINOS ND and FD fluxes (right). The weight in each matrix is the probability that a neutrino in detector A of energy $E_{a}$ will give a neutrino of energy $E_{b}$ in detector B. The point to notice is that there is a deal of asymmetry between the MINOS and $\mathrm{NO} \nu \mathrm{A}$ matrices which show differences as a function of energy on the source of the neutrinos. Clearly more work in this area is needed to fully understand the approach and the full reach of such studies.

\section{The NuMI Facility}

FNAL's NuMI Facility stands to be unique. With MINOS continuing to take data in the NuMI$\mathrm{NO} \nu \mathrm{A}$ beam, it delivers information unavailable elsewhere (intermediate energy neutrinos), along with the potential for an exceptionally well understood neutrino flux which could serve as an attractor for new neutrino experiments at a smaller scale. MINOS+ will provide new results during the period of 2013-2016, and will have the possibility to probe accurately unique physics beyond the reach of the off-axis experiments. The combination of MINOS+, NO $\nu \mathrm{A}$ and MINER $\nu \mathrm{A}$ will maintain FNAL in the position of a leading neutrino accelerator laboratory while LBNE gets completed. 


\section{Hardware}

The FD has been running since 2003. The scintillator light output has been dropping steadily over the years and at some point it will fall below threshold for tracking. Figure 19 shows the light level for the FD and ND detectors as a function of time. The ND is more adversely affected by the light level because it is only read out at one end. The effect of light loss on track reconstruction has been studied with cosmics. For the FD the reconstructed cosmic rate is flat to within $0.05 \%$ over the last 7 years while at the ND the loss of tracks is no larger than $0.25 \% /$ year. To get these numbers, percent level seasonal effects must be subtracted out, which works well at the FD but not so well at the ND. The estimate is that this loss rate in the ND is even lower with fiducial events (whose muon direction is optimal for the detector layout).

The temperature in the ND cavern has been fluctuating over the years between 23 and 27 degrees C (on average) between 2005 and 2011 and has risen about 2 degrees (on average) over that time. A higher temperature also results in a faster rate of reduction in light level. The new chiller is being installed in the ND cavern which will alleviate this worry. The temperature at the FD has been reasonably flat, averaging 21 degrees $\mathrm{C}$.

There is also some risk for both detectors that the company which fixes the DAQ back-end data aggregating cards will not retain this capability into the future. This is the most critical item, and some level of funding will be required to replenish the spares pool for all systems to keep the detectors running in this second, unanticipated, life.
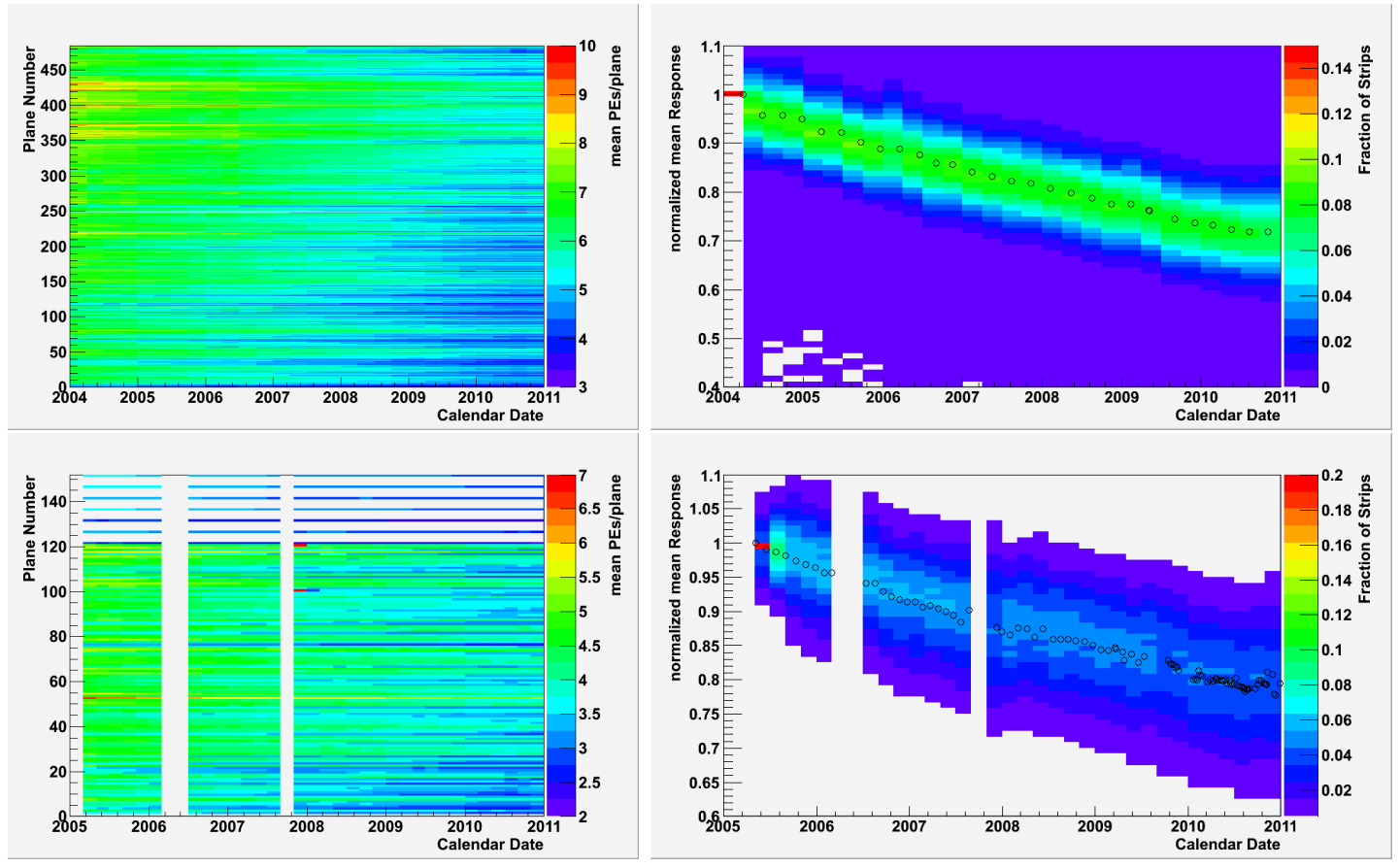

Figure 19: This plot shows the light level for the ND (upper) and FD (lower) as a function of time. Left is a scatter plot of the light level as a function of position in the detector and as a function of time, right is a profile plot showing the ratio of the light level compared to its original level with time. There is approximately twice as much light in the FD than the ND, owing to being read out at both ends. 
The full description of the hardware needed for the neutrino time of flight measurement is attached in Appendix E.

\section{$9 \quad$ Funding}

The timescale to finish MINOS was originally set to be the end of 2012. We are however already losing manpower to the other experiments. Such a new initiative will need to find extra funding for new people in the University groups because the proposal comes late and the groups have committed their future resources to existing new projects. The manpower is determined mostly by external sources, in particular the funding agencies. FNAL Directorate have communicated a short summary document to DOE, NSF and STFC (UK) for information purposes. The spokespeople have communicated with DoE (Alan Stone).

\subsection{Manpower Estimate}

Presently we have a number of University supported staff engaged in the MINOS running, calibration, processing, and data analysis. At least 25 people work at least half time on these tasks. There is additional manpower from the laboratory supported staff from BNL and FNAL which puts the total at 40 FTEs. While attempts will be made to further automate the processing, it is almost at the zero sum game position. We expect that about the same level of effort will be required to keep the detector up and running and the data being calibrated and processed and of course the physics analysis being carried out.

\subsection{Costs}

We estimate that keeping the skeleton mine crew at Soudan and the costs of the laboratory (shared with CDMS) will cost about 1M/yr. After CDMS moves to SNOLAB, this will fall entirely on the MINOS experiment. An estimated further $2 \mathrm{M} / \mathrm{yr}$ will be needed to cover postdocs and students in the universities (both NSF and DoE funded) for processing and analysis over the three year period. It is estimated that $\$ 350 \mathrm{~K}$ will be needed to stock the DAQ spares pool and an additional $\$ 350 \mathrm{~K}$ for the time of flight hardware. These costs notwithstanding, the beam and detector costs of over $170 \mathrm{M}$ dollars have already been invested, and the running costs of the beam are zero to MINOS+.

\section{Conclusion}

Continued running of MINOS in the NuMI-NO $\nu$ A beam will transform the FNAL NuMI program into a unique scientific complex, a facility which, when all experiments are working together, can deliver some of the best information about neutrinos to the world. Having a running experiment producing unique measurements will contribute to FNAL's continued leadership in neutrino physics during the MINOS-NO $\nu$ A-LBNE transition period and depending on the size of $\theta_{13}$, this experiment might be one of the only ways to get insight into the new (post 1999) field of neutrino physics. The MINOS+ experiment will be in a very fortunate position of being able to measure a green-field energy region where several rather disparate effects could reveal themselves. Such an opportunity comes very rarely, especially in such a relatively new field. 


\section{A Sterile Neutrino Mathematics}

\section{A.1 Reactor neutrino experiments}

Reactor neutrino experiments search for the disappearance of few-MeV electron neutrinos over baselines ranging from tens of metres to hundreds of kilometres.

From Eqn. 2, the general expression for the $\nu_{\mathrm{e}}$ survival probability in the case of four neutrino flavours is

$$
\begin{aligned}
P\left(\nu_{\mathrm{e}} \rightarrow \nu_{\mathrm{e}}\right)= & 1-4 \sum_{j>i}\left|U_{e j}\right|^{2}\left|U_{e i}\right|^{2} \sin ^{2} \Delta_{j i} \\
= & 1-4\left\{\left|U_{e 2}\right|^{2}\left|U_{e 1}\right|^{2} \sin ^{2} \Delta_{21}+\left|U_{e 3}\right|^{2}\left|U_{e 1}\right|^{2} \sin ^{2} \Delta_{31}\right. \\
& +\left|U_{e 4}\right|^{2}\left|U_{e 1}\right|^{2} \sin ^{2} \Delta_{41}+\left|U_{e 3}\right|^{2}\left|U_{e 2}\right|^{2} \sin ^{2} \Delta_{32} \\
& \left.+\left|U_{e 4}\right|^{2}\left|U_{e 2}\right|^{2} \sin ^{2} \Delta_{42}+\left|U_{e 4}\right|^{2}\left|U_{e 3}\right|^{2} \sin ^{2} \Delta_{43}\right\} .
\end{aligned}
$$

In the case that $m_{4} \gg m_{3}$, the three independent mass splittings differ from each other by orders of magnitude. Therefore the approximations $\Delta m_{31}^{2} \approx \Delta m_{32}^{2}$ and $\Delta m_{41}^{2} \approx \Delta m_{42}^{2} \approx \Delta m_{43}^{2}$ can be made. This allows the survival probability to be written

$$
\begin{aligned}
P\left(\nu_{\mathrm{e}} \rightarrow \nu_{\mathrm{e}}\right)= & 1-4\left\{\left|U_{e 2}\right|^{2}\left|U_{e 1}\right|^{2} \sin ^{2} \Delta_{21}\right. \\
& +\left|U_{e 3}\right|^{2}\left(\left|U_{e 1}\right|^{2}+\left|U_{e 2}\right|^{2}\right) \sin ^{2} \Delta_{31} \\
& \left.+\left|U_{e 4}\right|^{2}\left(\left|U_{e 1}\right|^{2}+\left|U_{e 2}\right|^{2}+\left|U_{e 3}\right|^{2}\right) \sin ^{2} \Delta_{41}\right\} .
\end{aligned}
$$

Using the following expressions for the PMNS matrix elements

$$
\begin{aligned}
& U_{e 1}=\cos \theta_{14} \cos \theta_{13} \cos \theta_{12}, \\
& U_{e 2}=\cos \theta_{14} \cos \theta_{13} \sin \theta_{12}, \\
& U_{e 3}=\cos \theta_{14} \sin \theta_{13}, \\
& U_{e 4}=\sin \theta_{14},
\end{aligned}
$$

the survival probability becomes

$$
\begin{aligned}
P\left(\nu_{\mathrm{e}} \rightarrow \nu_{\mathrm{e}}\right)= & 1-\cos ^{4} \theta_{14} \cos ^{4} \theta_{13} \sin ^{2}\left(2 \theta_{12}\right) \sin ^{2} \Delta_{21} \\
& -\cos ^{4} \theta_{14} \sin ^{2}\left(2 \theta_{13}\right) \sin ^{2} \Delta_{31} \\
& -\sin ^{2}\left(2 \theta_{14}\right) \sin ^{2} \Delta_{41} .
\end{aligned}
$$

The term in $\Delta_{21}$ is the oscillatory term probed by KamLAND. At the KamLAND energies and baseline, $\sin ^{2} \Delta_{31}$ and $\sin ^{2} \Delta_{41}$ average to $\frac{1}{2}$. Both a non-zero $\theta_{13}$ and a sterile neutrino (non-zero $\left.\theta_{14}\right)$ would produce an energy-independent $\nu_{\mathrm{e}}$ deficit in the KamLAND detector.

The term in $\Delta_{31}$ is the oscillatory term probed by CHOOZ, Double Chooz, Daya Bay, etc. At the energies and baselines of the short baseline reactor experiments, the first term in $\Delta_{21}$ can be neglected since, for such experiments, $\Delta_{21} \approx 0$.

\section{B Electron neutrino appearance in a muon neutrino beam}

The MiniBooNE and LSND experiments search for $\nu_{\mathrm{e}}$ appearance in a $\nu_{\mu}$ beam. The oscillation probability for such appearance is given by 


$$
\begin{aligned}
P\left(\nu_{\mu} \rightarrow \nu_{\mathrm{e}}\right)= & 4 \mathcal{R}\left\{\left|U_{\mu 3}\right|^{2}\left|U_{e 3}\right|^{2} \sin \Delta_{31}+\left|U_{\mu 4}\right|^{2}\left|U_{e 4}\right|^{2} \sin ^{2} \Delta_{41}\right. \\
& \left.+U_{\mu 4}^{*} U_{e 4} U_{\mu 3} U_{e 3}^{*}\left(\sin ^{2} \Delta_{31}-\sin ^{2} \Delta_{43}+\sin ^{2} \Delta_{41}\right)\right\} \\
& +2 \mathcal{I}\left\{U_{\mu 4}^{*} U_{e 4} U_{\mu 3} U_{e 3}^{*}\left(\sin 2 \Delta_{31}-\sin 2 \Delta_{41}+\sin 2 \Delta_{43}\right)\right\}
\end{aligned}
$$

where the relevant elements of the PMNS matrix are given by

$$
\begin{aligned}
U_{e 3} & =\cos \theta_{14} \sin \theta_{13} e^{i \delta_{1}}, \\
U_{e 4} & =\sin \theta_{14}, \\
U_{\mu 3} & =-\sin \theta_{14} \sin \theta_{13} e^{-i \delta_{1}} \sin \theta_{24} e^{-i \delta_{2}}+\cos \theta_{13} \sin \theta_{23} \cos \theta_{24}, \\
U_{\mu 4} & =\cos \theta_{14} \sin \theta_{24} e^{-i \delta_{2}} .
\end{aligned}
$$

Hence it can be seen that these experiments probe a mixture of $\theta_{14}$ and $\theta_{24}$.

\section{Disappearance of Neutral Current events}

The disappearance probability for neutral current (NC) events is given by $1-P\left(\nu_{\mu} \rightarrow \nu_{\mathrm{s}}\right)$, where

$$
\begin{aligned}
P\left(\nu_{\mu} \rightarrow \nu_{\mathrm{s}}\right)= & 4 \mathcal{R}\left\{\left|U_{\mu 3}\right|^{2}\left|U_{s 3}\right|^{2} \sin \Delta_{31}+\left|U_{\mu 4}\right|^{2}\left|U_{s 4}\right|^{2} \sin ^{2} \Delta_{41}\right. \\
& \left.+U_{\mu 4}^{*} U_{s 4} U_{\mu 3} U_{s 3}^{*}\left(\sin ^{2} \Delta_{31}-\sin ^{2} \Delta_{43}+\sin ^{2} \Delta_{41}\right)\right\} \\
& +2 \mathcal{I}\left\{U_{\mu 4}^{*} U_{s 4} U_{\mu 3} U_{s 3}^{*}\left(\sin 2 \Delta_{31}-\sin 2 \Delta_{41}+\sin 2 \Delta_{43}\right)\right\}
\end{aligned}
$$

The relevant elements of the PMNS matrix are given by

$$
\begin{aligned}
U_{\mu 3}= & -\sin \theta_{14} \sin \theta_{13} e^{-i \delta_{1}} \sin \theta_{24} e^{-i \delta_{2}}+\cos \theta_{13} \sin \theta_{23} \cos \theta_{24} \\
U_{\mu 4}= & \cos \theta_{14} \sin \theta_{24} e^{-i \delta_{2}} \\
U_{s 3}= & -\sin \theta_{14} \cos \theta_{24} \cos \theta_{34} \sin \theta_{13} e^{-i \delta_{1}} \\
& -\cos \theta_{13} \sin \theta_{23} \cos \theta_{34} \sin \theta_{24} e^{i \delta_{2}}-\cos \theta_{13} \cos \theta_{23} \sin \theta_{34}, \\
U_{s 4}= & \cos \theta_{14} \cos \theta_{24} \cos \theta_{34} .
\end{aligned}
$$

Hence the NC disappearance probability is sensitive to the angle $\theta_{34}$ (primarily through the $\sin \theta_{34}$ term in $\left.U_{s 3}\right)$ as well as to $\theta_{24}$. The $\theta_{14}$ dependence is minimal, since it only features through $\cos \theta_{14}$ which, for small $\theta_{14}$, is approximately unity.

\section{Non-standard Matter Interactions}

If neutrinos participate non-standard interactions, the flavor Hamiltonian will receive contributions from this effect similar to the standard matter interactions that give rise to the MSW effect [20,21]. In general NSI can be flavor changing or flavor conserving, with amplitudes proportional to the standard MSW matter effect $\epsilon_{\mu \mu} V, \epsilon_{\tau \tau} V$, and $\epsilon_{\mu \tau} V$, with $V=\sqrt{2} G_{F} N_{e}$. The limits on $\epsilon_{\mu \mu}$ are already stringent and the difference between $\bar{\nu}_{\mu}$ and $\nu_{\mu}$ oscillations is more sensitive to $\epsilon_{\mu \tau}$ than $\epsilon_{\tau \tau}[22]$, which is neglected in the following discussion. This gives the following NSI Hamiltonian.

$$
H_{\text {matter }}=\left(\begin{array}{cc}
0 & \epsilon_{\mu \tau} V \\
\epsilon_{\mu \tau}^{*} V & 0
\end{array}\right)
$$



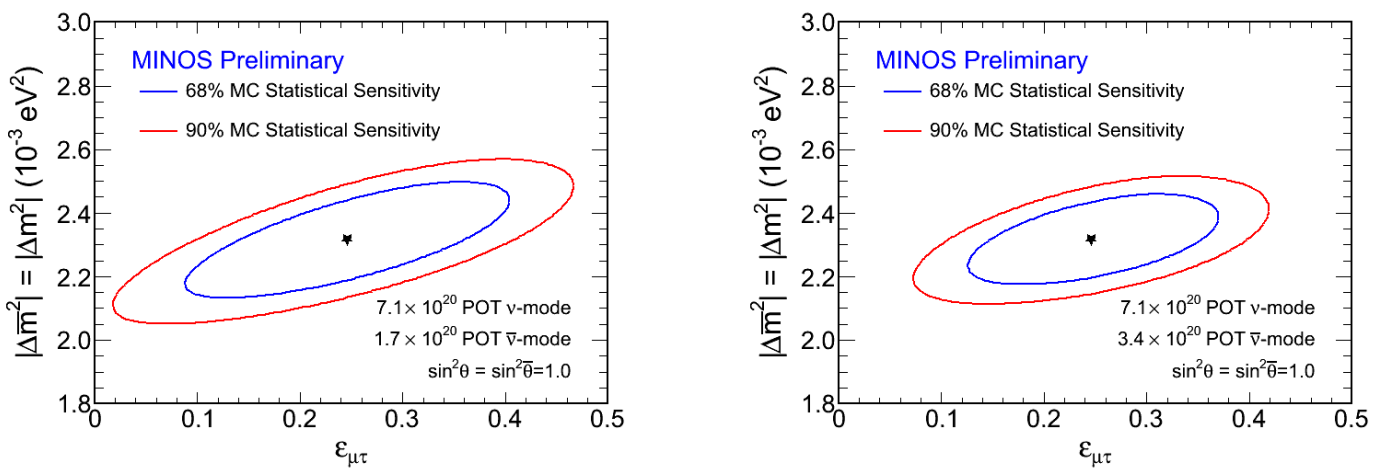

Figure 20: MINOS statistical sensitivity to non-standard interaction parameter $\varepsilon$ with existing data (left) and double the antineutrino-mode dataset.

Using the fact that $V \rightarrow-V$ for antineutrinos and taking $\epsilon_{\mu \tau}$ to be real we can write the full Hamiltonian as

$$
H=\left(\begin{array}{cc}
\sin ^{2} \theta_{23} \frac{\Delta m^{2}}{2 E} & \sin \theta_{23} \cos \theta_{23} \frac{\Delta m^{2}}{2 E} \pm \epsilon_{\mu \tau} V \\
\sin \theta_{23} \cos \theta_{23} \frac{\Delta m^{2}}{2 E} \pm \epsilon_{\mu \tau} V & \cos ^{2} \theta_{23} \frac{\Delta m^{2}}{2 E}
\end{array}\right)
$$

with which the survival probability in the case of maximal mixing $\left(\sin ^{2}(2 \theta)=1\right)$ becomes

$$
\begin{aligned}
P\left(\nu_{\mu}\right. & \left.\rightarrow \quad \nu_{\mu}\right) \simeq 1-\sin ^{2}\left(\frac{\Delta m_{23}^{2} L}{4 E} \pm \epsilon_{\mu \tau} V L\right) \\
& =1-\sin ^{2}\left(1.27 \frac{\Delta m^{2} L}{E} \pm \epsilon_{\mu \tau} V L\right)
\end{aligned}
$$

after restoring $\hbar$ and $c$.

In the more general case of non-maximal mixing

$$
P\left(\nu_{\mu} \rightarrow \nu_{\mu}\right)=1-\left(\frac{1.0-\cos ^{2} 2 \theta}{1 \mp 8 \sin 2 \theta \frac{\epsilon_{\mu \tau} V}{\Delta m_{23}^{2}} E+16\left(\frac{\epsilon_{\mu \tau} V}{\Delta m_{23}^{2}} E\right)^{2}}\right) \sin ^{2}\left(\frac{\Delta m_{23}^{2} L}{4 E} \pm \epsilon_{\mu \tau} V L\right) .
$$

MINOS sensitivity to the non-standard matter parameter $\epsilon_{\mu \tau}$ with existing low-energy neutrinoand antineutrino-mode data is shown in figure 20 .

Figure 21 shows the survival probability of neutrinos and antinetrinos in the presence of nonstandard interactions over the MINOS baseline as a function of energy. MINOS+ data can probe the $>5 \mathrm{GeV}$ region of this probability and improve the sensitivity to $\varepsilon$. 


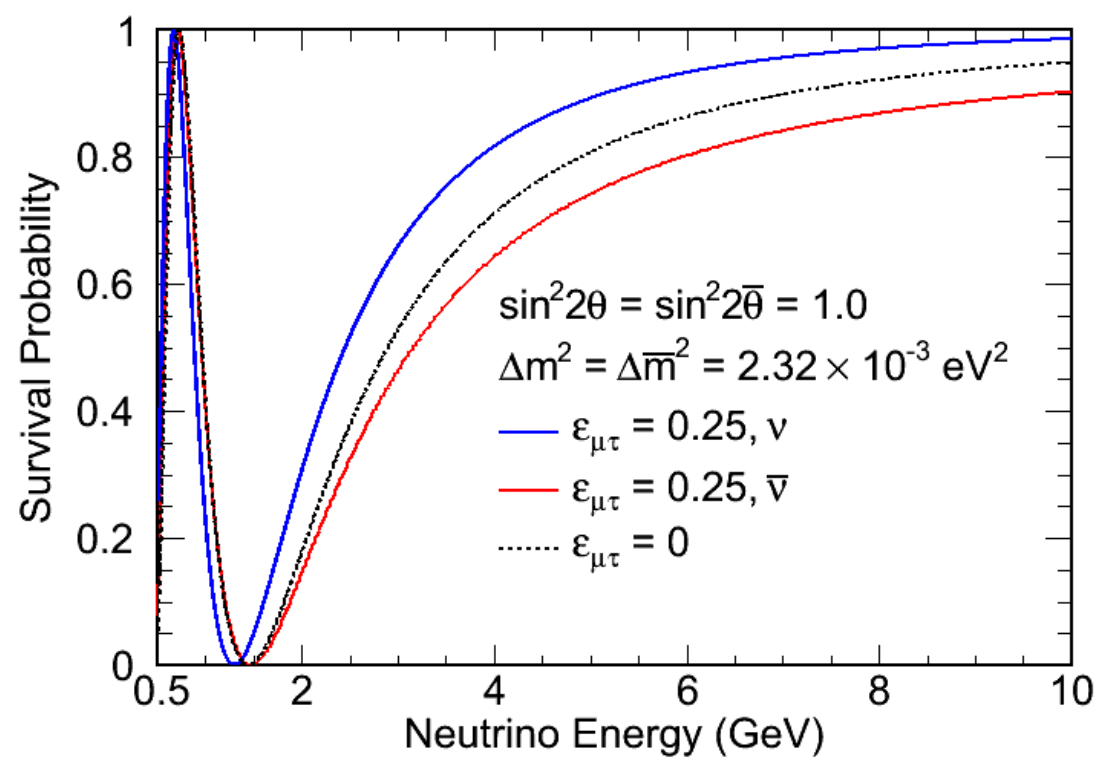

Figure 21: Survival probability of neutrinos and antineutrinos in the presence of non-standard matter interactions upon traveling $735 \mathrm{~km}$ from the source. MINOS best fit values for standard oscillations are assumed, $\Delta m_{23}^{2}=\Delta \bar{m}_{23}^{2}=2.32 \times 10^{-3} \mathrm{eV}^{2}$ and $\sin ^{2} 2 \theta_{23}=\sin ^{2} 2 \bar{\theta}_{23}=1.0$.

\section{E A Proposal to Measure the Speed of Muon Neutrinos to Five Parts in $10^{7}$ and Search for WIMP's in MINOS+}

In 2007, the MINOS collaboration conducted a measurement of of the neutrino velocity by comparing the detection time at the Near and Far detectors separated by $734 \mathrm{~km} \mathrm{[16].} \mathrm{The} \mathrm{precision}$ of the measured $\left|v_{\nu} / c-1\right|=(5.1 \pm 2.9) \times 10^{-5}$ was dominated by the systematic error on the time of flight which was 64 ns.

Here we propose to measure the propagation time of muon neutrinos $\left(\nu_{\mu}\right)$ from the NuMI source at Fermilab to the MINOS detector in northern Minnesota, a distance of $735.34 \mathrm{~km}$. The proposed timing instrumentation will provide an accuracy of $\simeq \pm 1 \mathrm{~ns}$ in the $\nu_{\mu}$ propagation time. With an accuracy in the distance of $\pm 0.1 \mathrm{~m}$, we can determine that the speed of a neutrino differs from the speed of light by no more than five parts in $10^{7}$. The time-of-flight instrumentation will also enable a sensitive search for slow-moving weakly-interacting particles produced in the NuMI beam. The proposed additional instrumentation will not perturb our progress toward oscillation physics, and should enrich the NuMI physics potential. The details of this idea are in presented in the MINOS-DocDB \#5046 [31]. 


\section{E.1 Motivation}

One of the neutrino beams constructed thirty years ago for the debut of Fermilab was the site of the first measurement of the speed of a neutrino [23]. The experiment showed for $\mu$-type neutrinos that $\left|v_{\nu} / c-1\right|<40 \times 10^{-6}$ [24]. Information on the speed of $e$-type neutrinos comes exclusively from the observation of neutrinos from SN1987A. Applying specific assumptions to the supernova data Longo [25] and Stodolsky [26] showed that $\left|v_{\nu} / c-1\right|<2 \times 10^{-9}$.

We propose here to undertake a measurement of the $\nu_{\mu}$ speed with precision considerably higher than achieved previously. The MINOS experiment, designed for the study of neutrino oscillations, offers an incomparable opportunity for this measurement. The $735 \mathrm{~km}$ distance between the production target and the Far Detector has been determined to an accuracy of $\pm 0.7 \mathrm{~m}$ [32], [33]. The dominant contribution to this error is the error in the separation between a benchmark on the earth's surface near the mine shaft and a benchmark in the MINOS cavern. The error was larger than anticipated, but at the time of the measurement it was deemed better than necessary for the purposes envisioned. With new objectives in mind we intend to remeasure this separation and thereby to reduce its error to less than $\pm 0.1 \mathrm{~m}$. With the proposed additional instrumentation to measure the propagation time, we can determine the $\nu_{\mu}$ speed to better than five parts in $10^{7}$.

The measurement that we propose, as good as it is, is not good enough to compete with conventional methods of constraining the $\nu_{\mu}$ mass. From analysis of charged pion decay we know that the mass of $\nu_{\mu}$ is less than $190 \mathrm{keV}$ [34]. The upper limit obtained from our speed measurement will at best be about $2 \mathrm{MeV}$, larger by a factor of 20 . Clearly we do not advocate this experiment as a mass measurement. Rather we regard the speed as an independent observable, and we seek to check that neutrinos behave as expected vis-à-vis this observable.

If the neutrino mass will not be under the proverbial microscope, are there less orthodox theoretical ideas that might be tested by a speed measurement? Chodos, Hauser, and Kosteleckỳ [35] proposed that the neutrino might be a tachyon, a faster-than-light object whose speed is inversely related to its energy. Hughes and Stephenson [36] criticized this idea, but were inturn rebuffed by Chodos et al. [37]. More recently several papers have proposed a limiting speed for neutrinos that is less than $c$ [27]. The time of flight measurement could also test for Lorentz invariance violation in neutrinos [28]. Tests of CPT violation [38] could also be made in the neutrino sector and compared to parameter limits from the photon sector. While we view these theoretical speculations as interesting, we do not regard them as essential to the motivation of the project. If neutrinos indeed adhere to some unorthodox theory, as likely as not it is a theory that no one has yet considered.

The Main Injector RF imposes a high-contrast microstructure on the NuMI beam, and this feature is essential to the speed measurement. We expect neutrinos to arrive at Soudan in phase with the RF buckets. As proposed by Shrock [39], the voids between buckets offer the complementary opportunity of a search for weakly-interacting particles that are massive, in our case $\geq 10 \mathrm{MeV}$. Gallas et al. conducted this kind of search within the confines of Fermilab [40]. They were sensitive to anomalous particles no lighter than $500 \mathrm{MeV}$.

One possible source of an anomalous weakly interacting particle, an "anomalon," is anomalous pion decay. The KARMEN experiment reported evidence for an anomalon from $\pi$ decay [41] that turned out to be an artifact. KARMEN data now rule out a particle matching their artifact, but an anomalon with slightly diferent properties remains consistent with their data and other data. One can also imagine that an exotic K decay channel or an exotic Primakoff effect in the interactions of

the primary protons on carbon produces the anomalon. The focusing of $\pi$ 's and K's by the NuMI 


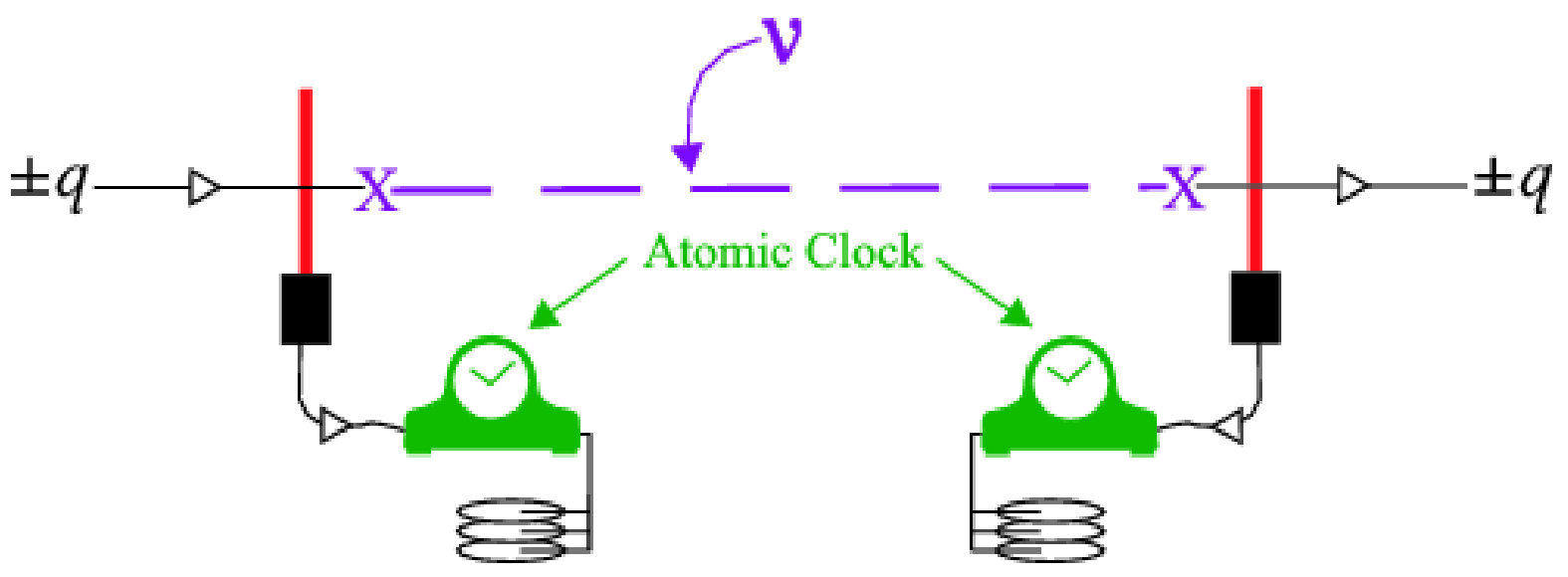

Figure 22: Method for measurement of time of flight using atomic clocks.

horns enhances the flux of their decay products in MINOS. Clearly the focusing does not enhance anomalons produced at the carbon target.

In the following we discuss methodology in the context of the $\nu_{\mu}$ speed measurement, but the identical technology and techniques enable the anomalous particle search. The two objectives will have equal claim on our interest and analysis effort.

The measurement of neutrino speed raises substantially the outreach potential of the MINOS project. The phenomenon of neutrino oscillation, the principal focus of study of NuMI-MINOS, is essentially quantum mechanical and is conceptually inaccessible to a majority of citizens. By contrast, virtually everyone appreciates the notion of speed. The measurement proposed here creates an opportunity to present to interested nonprofessionals a NuMI-MINOS activity that they can fully understand.

Our present estimate of the capital cost of this project is about $\$ 350 \mathrm{k}$ based upon 2005 -dollar. An important influence on the capital cost is the economics of communications satellite air time. We have designed around a source of air time that is economically satisfactory but not optimal for our application, and we are seeking a more compatible source. Success in this regard may reduce the capital cost by $\$ 36,000$.

\section{E.2 Methodology}

Conceptually a measurement of speed by time of flight is about as straightforward as a physical measurement can be. The conventional approach becomes unattractive, if not infeasible, when the source separation grows to hundreds of kilometers. Timekeeping with atomic clocks offers a viable alternative. In this approach we place one atomic clock (AC) at Fermilab and another in the cavern at Soudan as we show schematically in Fig. 22. The time of an arbitrary "event" at Fermilab is established by reference to the local AC and recorded locally and similarly for an event at Soudan. The delay between related events at the two locations may be determined by comparing the recorded clock times "offline."

The bunching of the proton beam imposed by the accelerator RF is the essential feature of the experiment that allows events at the two locations to be correlated. If we assume that the pions produced in the proton target travel at precisely $c$ and that the daughter neutrinos do likewise, then 


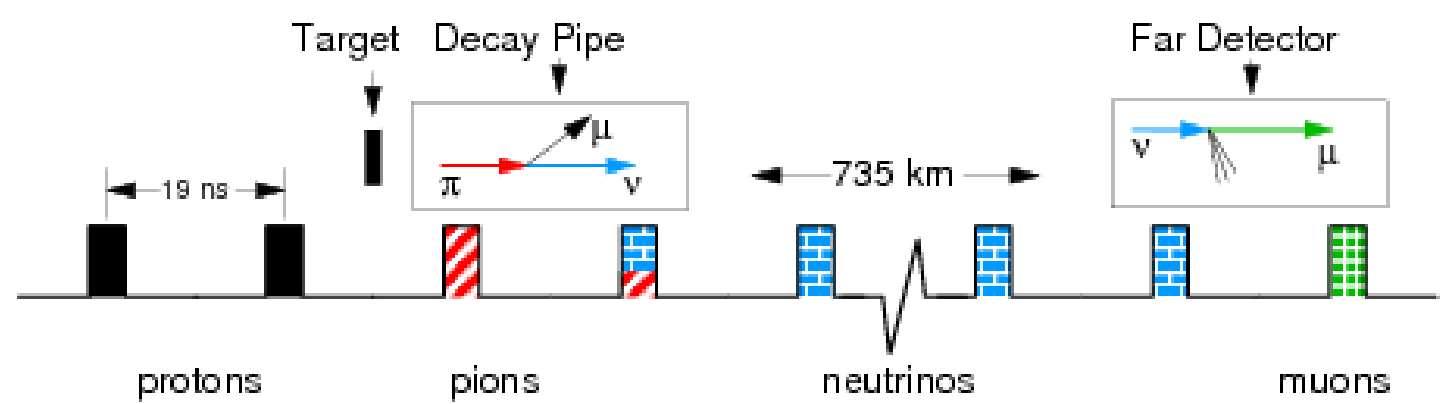

Figure 23: Between the Main Injector and Soudan the beam undergoes several metamorphoses but preserves the original microstructure.

the neutrinos arriving at Soudan will faithfully preserve the microstructure of the primary protons. This structure consists of pulses of width 2 ns spaced by 19 ns. In Fig. 23 we represent the various metamorphoses of the beam on its trip from the Main Injector to Soudan and the preservation of the pulse structure from beginning to end. Although pions actually propagate down the decay pipe at a speed a bit less than $c$, the delay induced is typically only $300 \mathrm{ps}$ for the neutrinos that we will catch in the Far Detector. We will measure the time of protons on target against the AC at Fermilab and the arrival time of a neutrino at Soudan against the AC in the cavern. Because we can not know from which bunch a neutrino was produced, and therefore we can know the transit time to Soudan only modulo the 19 ns period of the beam microstructure.

\section{E.3 Steps and Systematic Errors}

We enumerate the salient steps that will be undertaken to accomplish the proposed $\nu$-speed measurement and present the associated systematic errors.

(A) Distance from Fermilab to Soudan Site: The separation of benchmarks on the surface at Fermilab and at the Soudan site has already been determined to $\pm 1 \mathrm{~cm}$ [32]. The translation of the Soudan surface benchmark to the MINOS cavern incurs a larger error [33]. The error underground is currently estimated at $\pm 70 \mathrm{~cm}$. We consider this error to be acceptable for the first phase of this proposal. When we arrive eventually at the point that this error dominates the error budget of the speed measurement, we will remeasure the position of the benchmark in the cavern with an error not exceeding $\pm 10 \mathrm{~cm}$. The corresponding error in the $\nu$ time of flight (ToF) will be about $300 \mathrm{ps}$.

(B) Timekeeping and Time Transfer: A valid measurement of time of flight requires that we establish synchronization of the two clocks and maintain it while the neutrinos are in transit, an interval of about $2.5 \mathrm{~ms}$. The next challenge is to resynchronize the AC's on a schedule that holds the drift to less than 1.0 ns. The synchronization technology which will work is the two-way satellite time transfer (TWSTT). In practice the synchronization procedure is somewhat more elaborate. Most of the equipment, however, is available as a turn-key system [43]. We estimate this systematic error to be about $250 \mathrm{ps}$.

(C) Temporal Calibration of the Far Detector: The temporal calibration of the FD involves the integration of the AC's with the FD-electronics, a time model for the FD, and auxiliary calibration detectors composed of two sets of $1 \mathrm{~m} \times 1 \mathrm{~m}$ fast scintillating counters at the FD and ND locations. 
We do not foresee any reason that this error should exceed 300 ps.

(D) The Time of Protons on Target at Fermilab: A signal from the Main Injector RF will provide the time of protons on target, and the auxiliary detector at Fermilab will provide the calibration for this signal. The error on this will be entirely determined by the systematic accuracy with which the thick, fast scintillation counters, composing the auxiliary detector, measure the decay muons. We intend that the auxiliary detector should provide a single-event error for the arrival time of decay muons of about $1 \mathrm{~ns}$, and we anticipate that the resulting systematic error will be less than 300 ps.

(E) The Distance Traveled by the Parent $\pi^{+}$: A portion of the distance from the proton target at Fermilab to the Far Detector is travelled not by the neutrino but by the parent $\pi^{+}$. Our calculation of the average delay incurred by the $\pi^{+}$gives 300 ps. By simulation we will greatly refine this estimate, and only the residual error in the computation will remain as a systematic error.

In summary, we anticipate that none of the sources of systematic error will exceed $1 \mathrm{~ns}$, and we will have methods for controlling all of these uncertainties.

\section{E.4 Budget}

In the following tables we list the salient project costs.

\begin{tabular}{lcr}
$\begin{array}{l}\text { Capital costs: } \\
\text { Item }\end{array}$ & Quantity & \multicolumn{1}{c}{ Cost } \\
\hline \hline Agilent 5071A clock & 3 & $\$ 120 \mathrm{k}$ \\
Rubidium clock & 2 & $\$ 10 \mathrm{k}$ \\
TSC clock electronics & 2 & $50 \mathrm{k}$ \\
KU band modem & 2 & $36 \mathrm{k}$ \\
VSAT ground station & 2 & $30 \mathrm{k}$ \\
Radome & 2 & $32 \mathrm{k}$ \\
Auxiliary detector & 4 & $70 \mathrm{k}$ \\
\hline TOTAL & & $\$ 348 \mathrm{k}$
\end{tabular}

Annual operating expense:

\begin{tabular}{lcr} 
Item & Quantity & Cost \\
\hline \hline Air time & 300 days & $\$ 18 \mathrm{k}$ \\
Clock trips & 10 & $25 \mathrm{k}$ \\
\hline TOTAL & & $\$ 43 \mathrm{k}$
\end{tabular}

The auxiliary detector will be two sets of identical detectors placed at the near and the far sites. Each detector is composed of three planes of RPC's (or drift-chambers), to track muons, followed by two planes of fast scintillator planes. The transverse dimension of the components is $1 \mathrm{~m} \times 1 \mathrm{~m}$. Each plane is composed of $5 \mathrm{~cm} \times 1 \mathrm{~m}$ scintillator bars instrumented with fast photomultiplier tubes on either side. We propose to build one additional plane of tracker and scintillator-plane as spare. We estimate the cost of this system at $70 \mathrm{k} \$$ based upon prior experience of the South Carolina group with the construction of a time-of-flight (TOF) wall. The TOF wall was $3.5 \mathrm{~m} \times 3.5 \mathrm{~m}$ and 
yielded a precision of $\simeq 250$ ps for a precisely reconstructed charged track. The material cost of TOF was about $200 \mathrm{k} \$$. The present detector is about one third in size leading us to an estimate of $70 \mathrm{k} \$$.

In the table below we list salient project milestones in order of expected completion together with the expenditures required to attain the respective milestone. With timely funding, we expect to have the complete instrumentation ready within 12 months, and operational 3 months later. Carolina and Austin groups will lead the effort; we will also enlist the help of additional collaborators.

\begin{tabular}{lr} 
Milestone & $\begin{array}{r}\text { Integrated } \\
\text { cost } \\
\text { Dollars }\end{array}$ \\
\hline \hline Measure cosmic ray TOF using Rb & \\
clock and TSC electronics & $\$ 45 \mathrm{k}$ \\
Measure drift of Rb clocks & $35 \mathrm{k}$ \\
Synch Rb clocks using TWSTT & $126 \mathrm{k}$ \\
Two auxiliary detectors operational & $176 \mathrm{k}$ \\
Measure TOF at Fermilab & $208 \mathrm{k}$ \\
First attempt at MINOS Timing Model & $208 \mathrm{k}$ \\
Commission 2 Agilent 5071A clocks & $288 \mathrm{k}$ \\
Four auxiliary detectors operational & $308 \mathrm{k}$ \\
Relocate one clock ensemble and aux & \\
detectors to Soudan & $308 \mathrm{k}$ \\
First clock trips & $348 \mathrm{k}$
\end{tabular}




\section{References}

[1] J. Hosaka et al., Phys. Rev. D 74, 032002 (2006); B. Aharmim et al., Phys. Rev. C72, 055502 (2005); S. Abe et al., Phys. Rev. Lett. 100, 221803 (2008); M. H. Ahn et al., Phys. Rev. D74, 072003 (2006).

[2] P.Adamson et al., Phys. Rev. Lett. 106, No.18 (2011).

[3] P. Adamson et al., Phys. Rev. D81, 052004 (2010).

[4] B. Pontecorvo, JETP 34, 172(1958); V. N. Gribov and B. Pontecorvo, Phys. Lett. B28, 493 (1969); Z. Maki, M. Nakagawa, and S. Sakata, Prog. Theor. Phys. 28, 870 (1962).

[5] D. Michael et al., Nucl. Inst. \& Meth. A596, 190 (2008).

[6] V. D. Barger et al., Phys. Lett. B462, 109 (1999).

[7] E. Lisi, A. Marrone, and D. Montanino, Phys. Rev. Lett. 85, 1166 (2000).

[8] http://arxiv.org/abs/1104.0344

[9] Y. Ashie et al., Phys. Rev. Lett. 93, 101801 (2004); Y. Ashie et al., Phys. Rev. D71, 112005 (2005).

[10] $\mathrm{NO} \nu \mathrm{A}$ TDR

[11] P. Adamson et al., Phys. Rev. D82, 051102 (2010); M. Apollonio et al., Eur. Phys. J. C27, 331 (2003).

[12] P.A.N. Machado, H. Nunokawa,R. Zukanovich Funchal, hep-ph/1101.003v1.

[13] A.A. Aguilar-Arevalo et al., Phys. Rev. Lett. 105, 181801 (2010); Phys. Rev. Lett. 102, 101802 (2009); R. Van de Water, presentation at Neutrino 2010, Athens.

[14] A. Aguilar et al., Phys. Rev. D64, 112007 (2001).

[15] G.Mention et al.hep-ph/1101.2755v1.

[16] P. Adamson et al.,Phys. Rev. D76: 072005 (2007).

[17] C.D.Hoyle et al., Phys. Rev. Lett. 861418 (2001).

[18] J. Kopp, P.A.N. Machado and S.Parke, Phys.Rev.D82:113002 (2010).

[19] P. Adamson et al., Nucl. Instrum. Meth. A 566:119-113, 2006

[20] W. A. Mann and D. Cherdack, Oscillation $P\left(\bar{\nu}_{\mu} \rightarrow \bar{\nu}_{\mu}\right)$ is not like $P\left(\nu_{\mu} \rightarrow \nu_{\mu}\right)$ in the presence of an NSI $\epsilon_{\mu \tau}$ matter effect, MINOS-doc-6173 (2009)

[21] H. Minakata, Neutrinos' Non-Standard Interactions; another Eel under a Willow, arXiv:0905.1387[hep-ph]

[22] W. A. Mann et al., Apparent Multiple $\Delta m_{32}^{2}$ in $\bar{\nu}_{\mu}$ and $\nu_{\mu}$ Survival Oscillations from NonStandard Interaction Matter Effect, arXiv:1006.5720 (2010) 
[23] J. Alspector et al., Phys. Rev. Lett. 36, 837-840 (1976).

[24] G.R. Kalbfleisch et al., Phys. Rev. Lett 43,1361-1364 (1979).

[25] M.J. Longo, Phys. Rev. D36, 3276-3277 (1987).

[26] L. Stodolsky, Phys. Lett. B201, 353-354 (1988).

[27] S.C. Coleman and S.L. Glashow, Phy. Rev. D59, 116008 (1999). V. Ammosov and G. Volkov, hep-ph/0008032 (2000). G.S. Asanov, hep-ph/0009305 (2000).

[28] S. Choubey and S. King hep-ph/0311326 (2003).

[29] R.E. Schrock, Phys. Rev. Lett. 40,1688-1691 (1978).

[30] PoS (ICHEP 2010) 313

[31] S.R. Mishra and C. Rosenfeld, MINOS-DocDB \#5046.

[32] NuMI Alignment WBS 1.1.6 TDR Update, as of March 2001.

[33] J. Skaloud and K.-P. Schwartz, Zeitschrift für Vermessungswese 8, 292-299 (2000).

[34] K. Assamagan et al., Phys. Rev. D53:6065-6077 (1996).

[35] A. Chodos, A.I. Hauser, and V.A. Kosteleckỳ, Phys. Lett. B150:431-435 (1985).

[36] R.J. Hughes and G.J. Stephenson Jr., Phys. Lett. B244:95-100 (1990).

[37] A. Chodos et al., Mod. Phys. Lett. A 7, 467 (1992).

[38] D. Colladay and A. Kostelecky hep-ph/9703464 (1997).

[39] R.E. Schrock, Phys. Rev. Lett. 40:1688-1691 (1978).

[40] E. Gallas et al. (the FMMF Collaboration), Phys. Rev. D52:6-14 (1995).

[41] B. Armbruster et al. (the KARMEN Collaboration), Phys. Lett. B348:19-28 (1995). C. Oehler (the KARMEN Collaboration), Nucl. Phys. Proc. Supl. 85:101-104 (2000).

[42] V.S. Zhang, T.E. Parker, M.A. Weiss, and F.M. Vannicola, Proceedings of the IEEE/EIA International Frequency Control Symposium, June 7-9, 2000 , Kansas City, MO, pp. 598-606.

[43] Timing Solutions Corporation, <http://www.timingsolutions.com>.

[44] R. Lee and S. Seun, NuMI-L-694 (2000).

[45] <http://www.tm.agilent.com>.

[46] NuMI Baseline Beam Design Parameters, 7/16/01, $<$ http://www-numi.fnal.gov:8875/internal/beam/design_params.txt $>$. 